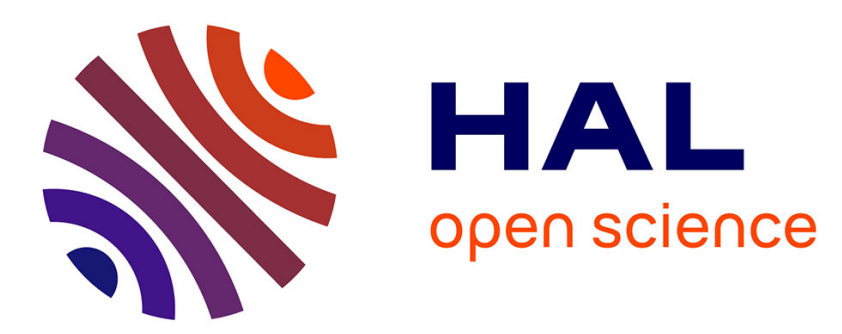

\title{
Nonlinear optimals in the asymptotic suction boundary layer: Transition thresholds and symmetry breaking
}

Stefania Cherubini, Pietro de Palma, Jean-Christophe Robinet

\section{To cite this version:}

Stefania Cherubini, Pietro de Palma, Jean-Christophe Robinet. Nonlinear optimals in the asymptotic suction boundary layer: Transition thresholds and symmetry breaking. Physics of Fluids, 2015, 27, pp.1.4916017. 10.1063/1.4916017 . hal-01212092

\section{HAL Id: hal-01212092 \\ https://hal.science/hal-01212092}

Submitted on 6 Oct 2015

HAL is a multi-disciplinary open access archive for the deposit and dissemination of scientific research documents, whether they are published or not. The documents may come from teaching and research institutions in France or abroad, or from public or private research centers.
L'archive ouverte pluridisciplinaire HAL, est destinée au dépôt et à la diffusion de documents scientifiques de niveau recherche, publiés ou non, émanant des établissements d'enseignement et de recherche français ou étrangers, des laboratoires publics ou privés. 


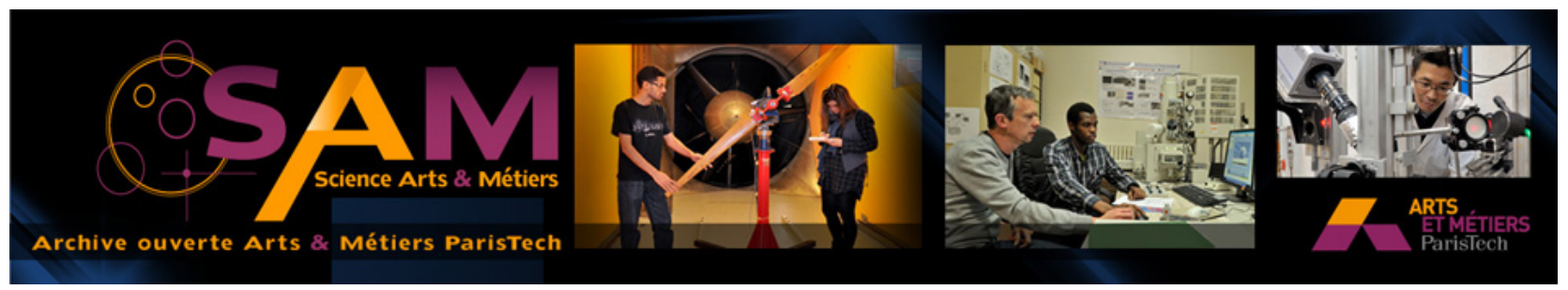

Science Arts \& Métiers (SAM)

is an open access repository that collects the work of Arts et Métiers ParisTech researchers and makes it freely available over the web where possible.

This is an author-deposited version published in: http://sam.ensam.eu

Handle ID: .http://hdl.handle.net/10985/10320

\section{To cite this version :}

Stefania CHERUBINI, Pietro DE PALMA, Jean-Christophe ROBINET - Nonlinear optimals in the asymptotic suction boundary layer: Transition thresholds and symmetry breaking - Physics of Fluids - Vol. 27, p.1.4916017 - 2015 


\title{
Non linear optimals in the asymptotic suction boundary layer: transition thresholds and symmetry breaking
}

\author{
S. Cherubini ${ }^{1}$, P. De Palma ${ }^{2}$, J.-Ch. Robinet ${ }^{1}$ \\ 1 DynFluid Laboratory, Arts et Metiers ParisTech, \\ 151, Bd. de l'Hopital, 75013 Paris, France \\ ${ }^{2}$ DMMM, CEMeC, Politecnico di Bari, \\ Via Re David 200, 70125 Bari, Italy
}

(Dated: August 16, 2014)

\begin{abstract}
The effect of a constant homogeneous suction on the non-linear transient growth of localized finite amplitude perturbations in a boundary-layer flow is investigated. Using a variational technique, non linear optimal disturbances are computed for the asymptotic suction boundary layer (ASBL) flow, defined as those finite amplitude disturbances yielding the largest energy growth at a given target time $T$. It is found that a strong enough wall suction remarkably reduces the optimal energy gain in the non linear case, and breaks the spanwise symmetry of the non-linear optimal perturbation found in the Blasius boundary-layer case. However, it appears that a value of the Reynolds number exists between 5000 and 10000 for which the non linear optimal perturbation changes from a non symmetric shape to a symmetric one. Direct numerical simulations show that the different structure of the base flow leads to a different evolution of the symmetric or non symmetric initial perturbation, due to the transport and tilting of the vortices by the mean flow. By bisecting the initial energy of the non linear optimal perturbations, minimal energy thresholds for subcritical transition to turbulence have been obtained. These energy thresholds are found to be 1 to 4 order of magnitude lower than the ones found by Levin et al. (2005) for other transition scenarios. For low to moderate Reynolds numbers, these thresholds are found to scale as $R e^{-2}$, suggesting a new scaling law for transition in the ASBL.
\end{abstract}

PACS numbers:

Keywords: 


\section{INTRODUCTION}

Drag reduction in external and internal flow is a fundamental topic in fluid mechanics since it is a key issue for improving the performance of engineering systems, increasing energy saving, and reducing environmental impact. Flow suction through the wall was among the first techniques applied to control the structure of the boundary layer in order to reduce drag. The idea, already employed by Prandtl and described in his first paper in 1904 [44], was born together with the concept of boundary layer itself, described for the first time in 1904 by Prandtl during the Third International Mathematics Congress at Heidelberg [1]. About thirty years later, such a technique was employed to delay transition over aircraft wings by reducing the boundary layer thickness and inducing a fuller velocity profile close to the wall [44]. The influence of suction on the stability of the boundary layer was studied by analytical methods considering uniform suction at wall. In particular, a very simple exponential solution for the velocity was derived by Meredith and Griffith (1938) [44] which would be valid at a sufficiently high distance from the leading edge of a flat plate. This solution of the Navier-Stokes equation is known as the asymptotic suction boundary layer (ASBL) [44] and is considered a suitable model to study boundary layers subject to active control by suction and to investigate the transition mechanism. Hocking (1975) [23] demonstrated that the critical Reynolds number is about two orders of magnitude higher than that of the Blasius boundary layer (BBL). In fact, the normal velocity term in the OrrSommerfeld and Squires equations stabilizes the Tollmien-Schlichting (TS) waves, producing an effective way of damping their asymptotic growth. This effect has been widely employed in different forms in the design of aircraft wing (see, for example, Joslin (1998) [26]).

More recently, the development of the optimal transient growth analysis has renewed the interest in the study of the ASBL. In fact, it is well known that for a sufficiently high level of free-stream turbulence (FST), a bypass route to transition may occur in the boundary layer which corresponds to the growth of linear optimal perturbations (LOP) [19, 37]. This mechanism is based on the development of streamwise-aligned structures composed by alternating low and high velocity streaks observed for the first time by Klebanoff [30]. The algebraic growth of the streaks due to the lift-up effect [32] leads eventually to secondary instability and break-up to turbulence [3, 4, 24]. From a numerical point of view, LOP were computed for several shear flows $[2,5,15,35,43]$. In all of these works, optimal 
perturbations are defined as those initial flow states yielding the largest amplification of the disturbance energy over a time/space interval and can be computed using a variational optimization approach $[18,36]$. For the case of the boundary layer at low Reynolds number, such optimal structures consists of pairs of streamwise aligned counter-rotating vortices producing streamwise streaks by the lift-up effect, in perfect agreement with the above experimental findings. The same mechanisms have been studied in the ASBL. Fransson and Alfredsson (2003) [20] performed an experimental analysis about the development of forced TS waves and about the algebraic growth of disturbances induced by free-stream turbulence. They confirmed the damping of TS waves due to suction and were able to suppress transition in both cases. Using a local approach, Fransson and Corbett (2003) [21] computed LOP for the ASBL and compared their results with experiments. They observed a significant transient growth, although smaller than in the case of the BBL. This indicates that the strong effect of the damping of the energy growth of TS waves obtained by suction is not achieved in the case of the algebraic growth. Bystrom et al. (2007) [6] computed LOP for the semi-suction boundary layer in order to take into account the presence of a small region free of suction close to the leading edge of the flat plate. With this approach, they could improve the agreement of the results with experimental data, demonstrating that the optimal energy growth is indeed obtained in the upstream region without suction. Finally, Levin et al. (2005) [33] studied the energy thresholds for transition to turbulence in the ASBL, for $R e=500,800$, 1200, with perturbations having the form of oblique waves, streamwise vortices, or random noise; whereas, Levin et al. (2007) [34] analyzed the energy threshold for the same Reynolds numbers, in the case of localized disturbances, and investigated the formation and evolution of turbulent spots.

The ASBL has been also considered for testing and validating the recent dynamical system theory of turbulence, which analyze the fundamental role of non linearities in transition to and sustainment of turbulence for shear flows. Such a theory relies on: 1) the observation of the existence of exact coherent states, which can be unstable fixed points, periodic orbits or chaotic solutions of the Navier-Stokes equations, having a few unstable directions (see Ref. $[17,25,39,50,52,53]) ; 2)$ the idea that such states constitute the skeleton of transition and regeneration processes of turbulence and can be used to understand its nature. In particular, for analyzing the transition process, it is interesting to study those flow perturbations confined on the boundary between the laminar and the turbulent states, called the edge of 
chaos $[9,16,45,48]$. Those perturbations can be very dangerous, being the closest ones to the laminar state capable of triggering transition. On the edge of chaos, one or more relative attractors may live, called edge states [48], which can be fixed points [46], periodic orbits [31] or chaotic states [17]. Concerning the ASBL, Kreilos et al. (2013) [31] investigated the structure of the edge-states for turbulence transition, identifying a periodic orbit embedded in the laminar-turbulent boundary. Such a solution shows the same basic mechanisms of transient growth interactions between streamwise-aligned vortices and streaks which characterize many shear flows. Furthermore, it captures also the bursting phenomenon typical of the BBL. Khapko et al. [28, 29] have investigated the dynamics restricted to the laminarturbulent boundary, describing the complex spatio-temporal dynamics of different localized edge states for several streamwise wavelength. They found that all these states have the same structure, consisting of a localised pair of low- and high-speed streaks flanked with streamwise vortices. Investigating the structure of the relative attractors embedded in the edge of chaos allows to identify the typical shape and dynamics of the coherent structures consituting the skeleton of turbulence. However, for unraveling the main features of the most effective (in terms of both time and energy) path to transition, the minimal-energy states on the laminar-turbulent boundary should be analyzed [7, 42].

Very recently, the problem of finding the minimal energy perturbation on the edge of turbulence has been investigated by solving the non linear optimal growth problem for finiteamplitude initial perturbations (see [27] for a review). Those perturbations which optimize at a given (target) time the growth of a functional linked to transition (the kinetic energy or the dissipation for instance), called non linear optimal perturbations (NLOPs), have been found for a pipe flow [40, 41]; a boundary layer flow [8, 10]; and a Couette flow [12, 16, 38, 42]. By optimizing the energy at large target times and bisecting the initial energy to bring the perturbation close to the laminar-turbulent boundary, the perturbation of minimal energy capable of bringing the flow to the edge state and then to transition, called the minimal seed of turbulent transition can be found [42]. When small target times are considered, a different procedure should be used for finding minimal-energy perturbations on the edge of chaos, directly targeting the neighbourhood of the edge state in a finite time [7].

In all cases, the NLOPs are characterized by a very different structure with respect to the linear optimal ones and largely outgrow them in energy due to non linear mechanisms [10, 41]. For the boundary-layer and the Couette flow, the NLOPs are characterized by a similar 
fundamental structure, composed of a localized array of vortices and low-momentum regions of typical length scale, capable of maximizing the energy growth most rapidly. Cherubini et al. (2011) [10, 11] have discussed the contribution of non linear effects in such a strong energy growth, showing that non linearity is crucial to sustain the growth of such optimal perturbations. The knowledge of these non-linear mechanisms may allow one to design effective control strategies to delay transition by using wall suction [14]. The aim of the present paper is to extend the analysis of the NLOP to the case of the ASBL, following the approach that the authors have employed for the BBL, discussing similarities and differences between these two cases, and highlighting the role of the suction velocity.

The paper is organized as follows. In the second section we define the problem and describe the non linear optimization method. In the third section, divided into three parts, a thorough discussion of the results of the non linear optimization analysis is provided. In particular, in the first part, the focus is on the characterization of the NLOP with respect to the LOP, also by comparing with the results already obtained for the BBL and Couette flows. The second part provides an analysis varying the Reynolds number, achieving supercritical values; whereas, the third part deals with the optimal route to turbulence, computed by the DNS. Finally, concluding remarks are provided.

\section{PROBLEM FORMULATION}

\section{A. Governing equations and numerical method}

The behaviour of an incompressible flow is governed by the Navier-Stokes (NS) equations:

$$
\begin{aligned}
& \frac{\partial \mathbf{u}}{\partial t}+(\mathbf{u} \cdot \nabla) \mathbf{u}=-\nabla p+\frac{1}{R e} \nabla^{2} \mathbf{u} \\
& \nabla \cdot \mathbf{u}=0
\end{aligned}
$$

where $\mathbf{u}=(u, v, w)$ is the velocity vector and $p$ indicates the pressure term. Dimensionless variables are defined with respect to the inflow boundary-layer displacement thickness $\delta^{*}$ and the freestream velocity, $U_{\infty}$, so that the Reynolds number is $R e=U_{\infty} \delta^{*} / \nu, \nu$ being the kinematic viscosity. A Cartesian coordinate system is considered, $x, y$ and $z$ being the streamwise, wall-normal and spanwise directions, respectively. The asymptotic suction boundary-layer flow is defined as the flow over a flat plate with a uniform wall-normal suction 
velocity $V_{S}=1 / R e$ applied along the wall. Therefore, imposing no-slip conditions for the streamwise and spanwise components of the velocity at the wall, one obtains from equations (1) the following solution [22]:

$$
\mathbf{U}=\left(\left(1-e^{-y}\right),-V_{S}, 0\right)^{T} .
$$

The numerical simulations are performed by integrating the NS equations with the following boundary conditions: at the bottom boundary, no-slip conditions for the $x$ and $z$ components of the velocity and homogeneous suction for the $y$ component, $v=-V_{S}$; at the upper boundary, the $z$ component of the velocity and of the vorticity are set to zero and homogeneous injection is imposed for the $y$ component of the velocity; in the streamwise and spanwise directions, periodicity is imposed for the three velocity components.

The analysis has been performed using five values of the Reynolds number (see table I), obtained varying the suction velocity $V_{S}$. The NS equations are discretized by a finitedifference fractional-step method using a staggered grid [49]. A second-order-accurate centered space discretization is used. Performing a grid-convergence analysis, a mesh made up by $451 \times 100 \times 61$ points has been selected for the reference domain at $R e=610$ with dimensions $L_{x}=100, L_{y}=20$ and $L_{z}=10.5$. The spanwise dimension has been chosen very close to the one used in [33] for determining transition thresholds, whereas the streamwise length is much longer to avoid interaction of the flow structures with its own tail for long target times. Since the NLOP has been found to localize more and more with increasing Reynolds number, the domain length has been reduced accordingly, in order to reduce the computational cost. Table I summarizes the domain lengths and the corresponding grid points selected for different Reynolds numbers after validations with respect to larger domains.

\section{B. Non linear optimization}

The non linear behavior of a perturbation $\mathbf{q}=\left(u^{\prime}, v^{\prime}, w^{\prime}, p^{\prime}\right)^{T}$ evolving in the laminar asymptotic suction boundary-layer flow is analyzed by solving the NS equations written in perturbative formulation with respect to the steady state solution, $\mathbf{Q}=(\mathbf{U}, P)^{T}$, with $\mathbf{U}$ given by equation (2). A zero perturbation boundary condition is imposed for the three velocity components at the $y$-constant boundaries, whereas periodicity of the perturbation is forced in the spanwise and streamwise directions. 


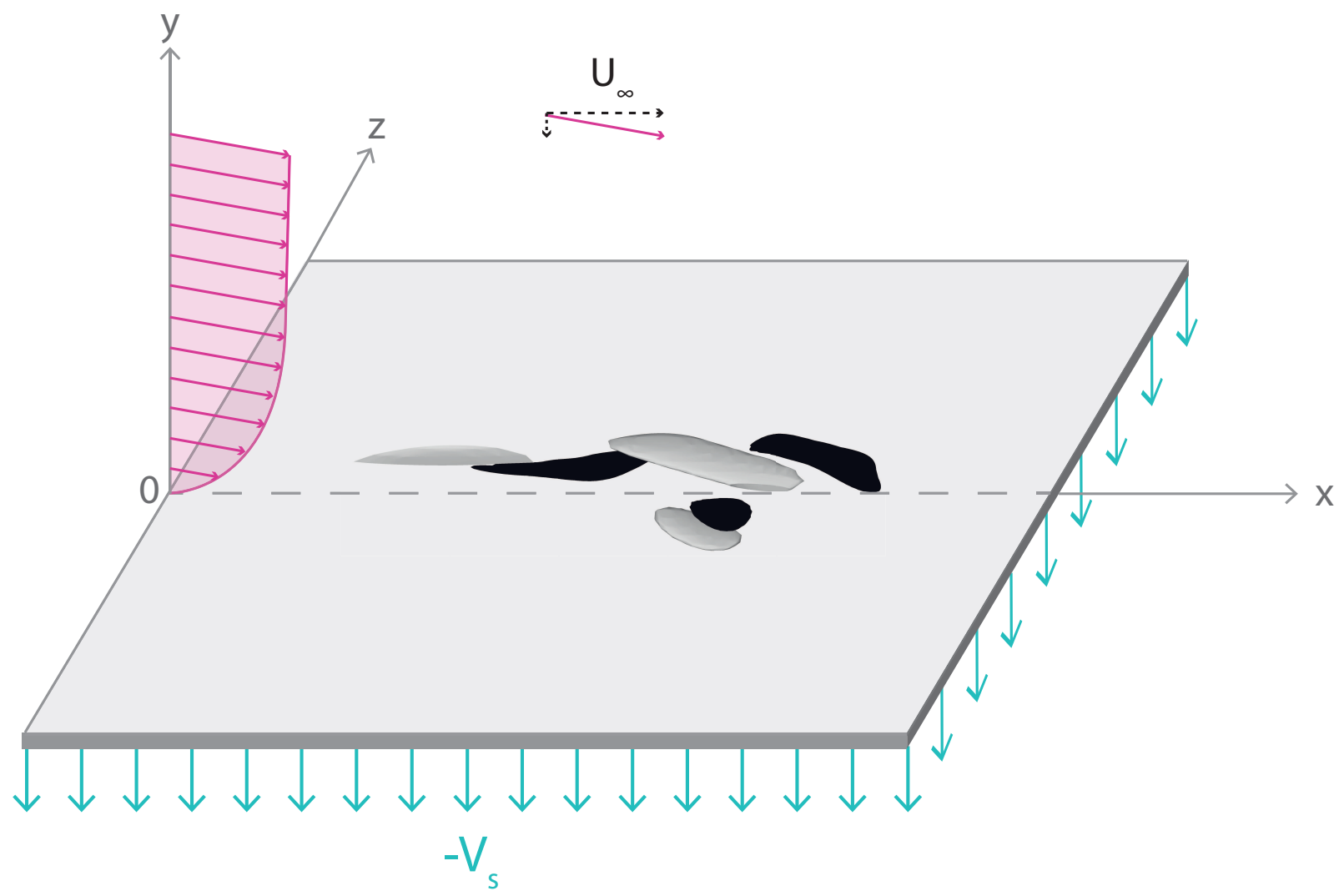

Figure 1: Sketch of the ASBL flow with a superposed localized disturbance.

\begin{tabular}{cccc}
\hline Re & $L_{x} \times L_{y} \times L_{z}$ & $N_{x} \times N_{y} \times N_{z}$ & $\mathrm{~T}$ \\
\hline 610 & $100 \times 20 \times 10.5$ & $451 \times 101 \times 61$ & $50,100,150,200,250$ \\
1200 & $50 \times 15 \times 7$ & $451 \times 101 \times 61$ & $50,100,150$ \\
2500 & $40 \times 15 \times 5$ & $451 \times 101 \times 61$ & $50,100,150$ \\
5000 & $40 \times 7.5 \times 4$ & $451 \times 101 \times 91$ & $50,100,150$ \\
10000 & $30 \times 7.5 \times 2$ & $601 \times 101 \times 91$ & 50,100 \\
\hline
\end{tabular}

Table I: Domain lengths, grid points and target times chosen for the optimizations at different Reynolds numbers

The goal is to find the perturbation at $t=0$ providing the largest disturbance growth at a given target time, $T$. At this purpose, a Lagrange multiplier technique is used $[8,36,40,55]$ to perform a constrained optimization of the perturbation energy. The disturbance energy 
density is defined as

$$
E(t)=\frac{1}{2 V} \int_{V}\left[u^{\prime 2}(t)+v^{\prime 2}(t)+w^{\prime 2}(t)\right] d V=\frac{1}{2 V}\left\langle\mathbf{u}^{\prime}(t) \cdot \mathbf{u}^{\prime}(t)\right\rangle
$$

where $V$ is the volume of the computational domain. Given an initial energy $E(0)=E_{0}$, we aim at finding the shape and amplitude of an initial perturbation $\mathbf{q}_{0}$ which induces at target time $T$ the largest energy gain $E(T) / E_{0}$; threfore, the objective function of the optimization procedure is $\Im=E(T) / E(0)$. The Lagrange multiplier technique consists in searching for extrema of an augmented functional, $\mathcal{L}$, with respect to every independent variable, the three-dimensional incompressible NS equations and the value of the initial energy being imposed as constraints. The augmented functional reads:

$$
\begin{aligned}
& \mathcal{L}=\frac{E(T)}{E(0)}-\int_{0}^{T}\left\langle\mathbf{u}^{\dagger} \cdot\left\{\frac{\partial \mathbf{u}^{\prime}}{\partial t}-\mathbf{u}^{\prime} \cdot \nabla \mathbf{U}+\mathbf{U} \cdot \nabla \mathbf{u}^{\prime}+\mathbf{u} \cdot \nabla \mathbf{u}^{\prime}-\nabla p^{\prime}-\frac{\nabla^{2} \mathbf{u}^{\prime}}{R e}\right\}\right\rangle d t \\
& -\int_{0}^{T}\left\langle p^{\dagger} \cdot \nabla \mathbf{u}^{\prime}\right\rangle d t-\lambda\left(\frac{E_{0}}{E(0)}-1\right) .
\end{aligned}
$$

where $\left(\mathbf{u}^{\dagger}, p^{\dagger}, \lambda\right)$ are the Lagrange multipliers, e.g. the adjoint variables. Integrating by parts and setting to zero the first variation of $\mathcal{L}$ with respect to $\left(\mathbf{u}^{\prime}, p^{\prime}\right)$ leads to the adjoint equations plus the compatibility condition (which are provided in Ref. [10]). The adjoint equations are linked to the direct ones by the presence of the direct variables in the advection terms, so that the whole flow field needs to be stored at each time step, requiring a remarkable storage capacity. The gradient of the augmented functional with respect to the initial perturbation $\mathbf{q}_{0}$ is forced to vanish by means of a conjugate gradient algorithm as detailed in Ref. [10]. A coupled iterative approach similar to that used in [55] and [40] is used to solve the problem, relying on the forward and backward solution of the direct and adjoint NS equations, respectively, and on the update of the initial perturbation in the conjugate gradient direction at each iteration, until convergence is reached. A detailed description of the optimization technique and of its convergence properties is provided in Ref. [10] for the case of the BBL flow and in Ref. [12] for the Couette flow.

\section{RESULTS}

\section{A. Non linear optimal perturbations at $R e=610$}

The non linear optimization has been performed at Reynolds number $R e=610$. This rather low Reynolds number (compared to the critical one for the ASBL) has been chosen 


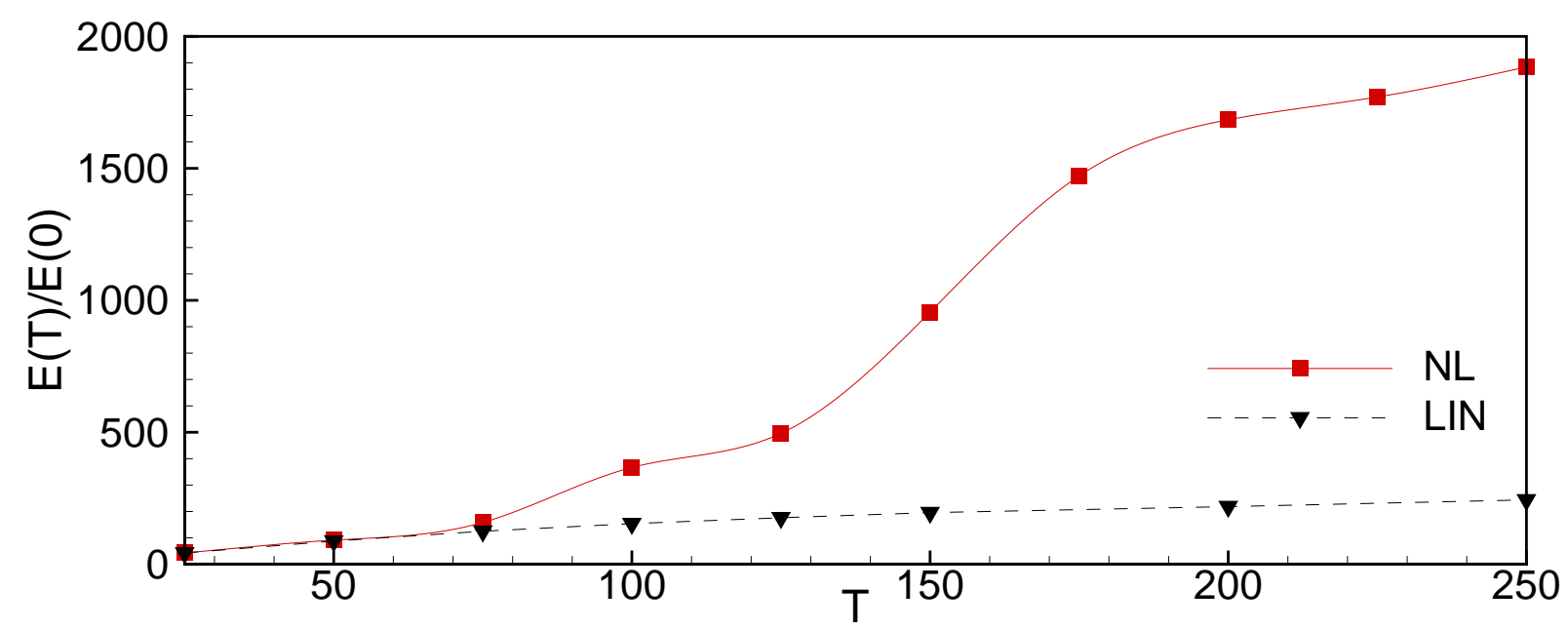

Figure 2: (Color online) Optimal energy gain versus target time $T$ for $R e=610, E_{0}=3.0 \times 10^{-7}$. The dashed line with triangles indicates the results of the linear optimization; the solid line with squares (red online) indicates the results of the non linear optimization.

for comparison purpose with the BBL case of ref. [8]. Figure 2 shows the value of the optimal energy gain versus the target time for an initial energy $E_{0}=3.0 \times 10^{-7}$. The dashed line refers to the results of a linear optimization, whereas the solid line represent the non linear optimization. As also observed for the BBL flow [8], the non linear optimal energy gain is remarkably larger than the corresponding linear one for $T>50$. The influence of the parameter $E_{0}$ on the value of the optimal energy is shown in Figure 3, for three values of the target time. It appears that a non linearity threshold value of the initial energy exists from which strong differences are observed in the non linear optimal energy with respect to the linear one (compare the solid lines with the dashed ones). Such a threshold decreases when the target time increases, as one can observe by comparing the solid lines in Figure 3 , converging towards a value, $E_{0}=1.2 \times 10^{-7}$, which might be close to the energy of the minimal seed for this Reynolds number (i.e., the perturbation of minimal energy on the laminar-turbulent boundary). Table II provides a comparison between the energy gains obtained for the BBL and the ASBL at $T=75$ (the behavior is similar for different target times) for three optimizations: a linear optimization and two non linear optimizations with $E_{0}=1.2 \times 10^{-7}$ and $E_{0}=3.0 \times 10^{-7}$, respectively. The results indicate that a significant reduction of the optimal energy growth is obtained in the linear case; however, wall suction 


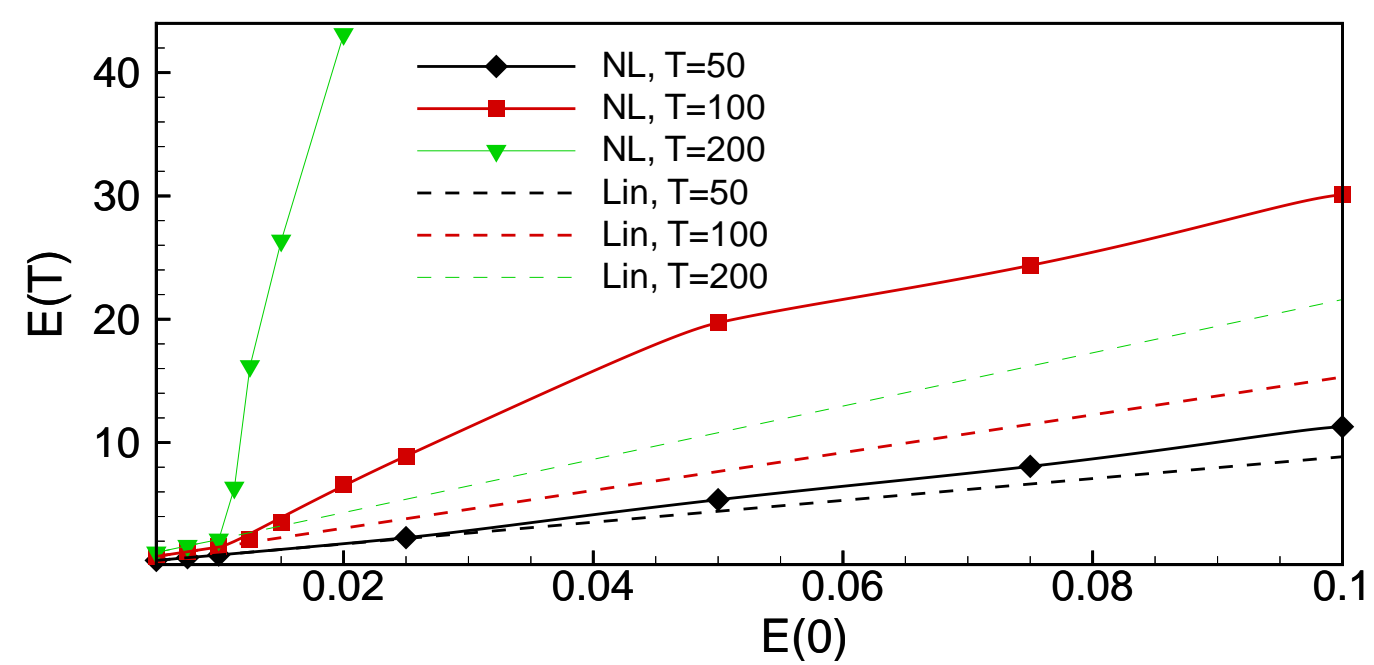

Figure 3: (Color online) Optimal energy for $R e=610$ at target time $T=50$ (black), $T=100$ (red), and $T=200$ (green) versus the initial energy $E(0)$, using the non linear optimization (solid lines with symbols) and the linear optimization (dashed lines).

\begin{tabular}{cccc}
\hline Test case & Linear & $E_{0}=1.2 \times 10^{-7}$ & $E_{0}=3.0 \times 10^{-7}$ \\
BBL & 275.10 & 801.98 & 1104.1 \\
ASBL & 125.16 & 125.15 & 158.37 \\
\hline
\end{tabular}

Table II: Comparison between energy gains at $T=75$ for the BBL and the ASBL.

is much more effective in damping the growth of non linear optimal perturbations.

Crossing the non linearity threshold also yields large modifications in the shape of the optimal perturbations. This can be observed in Figure 4, which provides the optimal initial perturbations obtained for the ASBL at $R e=610$ and $T=75$, for two values of the initial energy, $E_{0}$. For the lowest one, $E_{0}=1.2 \times 10^{-7}$ (top frame), the perturbation is similar to that obtained by the linear optimization in a BBL flow [13], being characterized by alternated vortices elongated in the streamwise direction (black and white surfaces), localized in two different positions along the flat plate. Due to weak non linear effects, which are non-negligible for such values of the initial energy, some spanwise modulations are present on the streamwise perturbation (green surfaces). Concerning the amplitudes, the largest perturbation velocity component is the spanwise one $\left(\left|w_{\max }\right|=0.0027\right)$, followed by the wall-normal $\left(\left|v_{\max }\right|=0.0025\right)$ and the streamwise one $\left(\left|u_{\max }\right|=0.0003\right)$. One can notice 
that the streamwise perturbation is one order of magnitude lower than the others, meaning that for this value of the initial energy the mechanism of growth is still very close to the linear optimal one, based on the lift-up of the streamwise base flow velocity by the vortices given by the wall-normal and spanwise perturbation. However, as one can observe in figure 4, the shape of the optimal perturbation changes remarkably between $E_{0}=1.2 \times 10^{-7}$ and $E_{0}=3.0 \times 10^{-7}$. The most striking difference is the strong localization of the disturbance in both the streamwise and spanwise direction. In fact, for initial energies larger than the non linearity threshold, a strong localization of the initial perturbation leads to larger amplitudes (for the same initial energy), triggering non linear effects that induce a remarkable increase of the energy gain at target time. In fact, for an increase of the initial energy of a factor 2.5, we observe at $t=0$ an increase of the velocity magnitudes of a factor of about 12 for $v$ and $w$, whereas a factor 80 is obtained for $u$. These values of the perturbation velocity components, together with the particular shape of the disturbance, are able to trigger non linear effects which allow a much larger energy growth than in the linear case.

This strong localization appears to be a typical feature of NLOP in shear flows, since it has been also observed for the pipe [40, 41], the BBL [10], and the Couette flow [12, 38, 42]. Furthermore, not only the extension, but also the structure of the perturbation changes remarkably. For $E_{0}=3.0 \times 10^{-7}$, the optimal perturbation is composed by three streamwisealternated vortices showing a finite inclination with respect to the streamwise direction (black and white surfaces), whereas in the quasi-linear case at $E_{0}=1.2 \times 10^{-7}$ the vortices are streamwise-aligned. On both flanks of such inclined vortices, localized patches of finiteamplitude streamwise disturbance are observed (green surfaces). Concerning the relative magnitude of the velocity perturbations, the largest perturbation velocity component is the spanwise one $\left(\left|w_{\max }\right|=0.033\right)$, followed by the streamwise $\left(\left|u_{\max }\right|=0.03\right)$ and the wallnormal one $\left(\left|v_{\max }\right|=0.024\right)$. These values are similar to those found for the Couette flow [12], whereas, for the BBL flow at the same Re, the largest component has been found to be the streamwise one, whose value is about half of the maximum value found here for the ASBL, for an initial energy just above the non linearity threshold, see [10]. It is worth noticing that, for all of these flows, in the linear case the streamwise velocity component at initial time is from one to two orders of magnitude lower than the spanwise and the streamwise ones, whereas in the non linear case all of the components are of the same order, meaning that different mechanisms are responsible for the growth of the perturbation energy. 


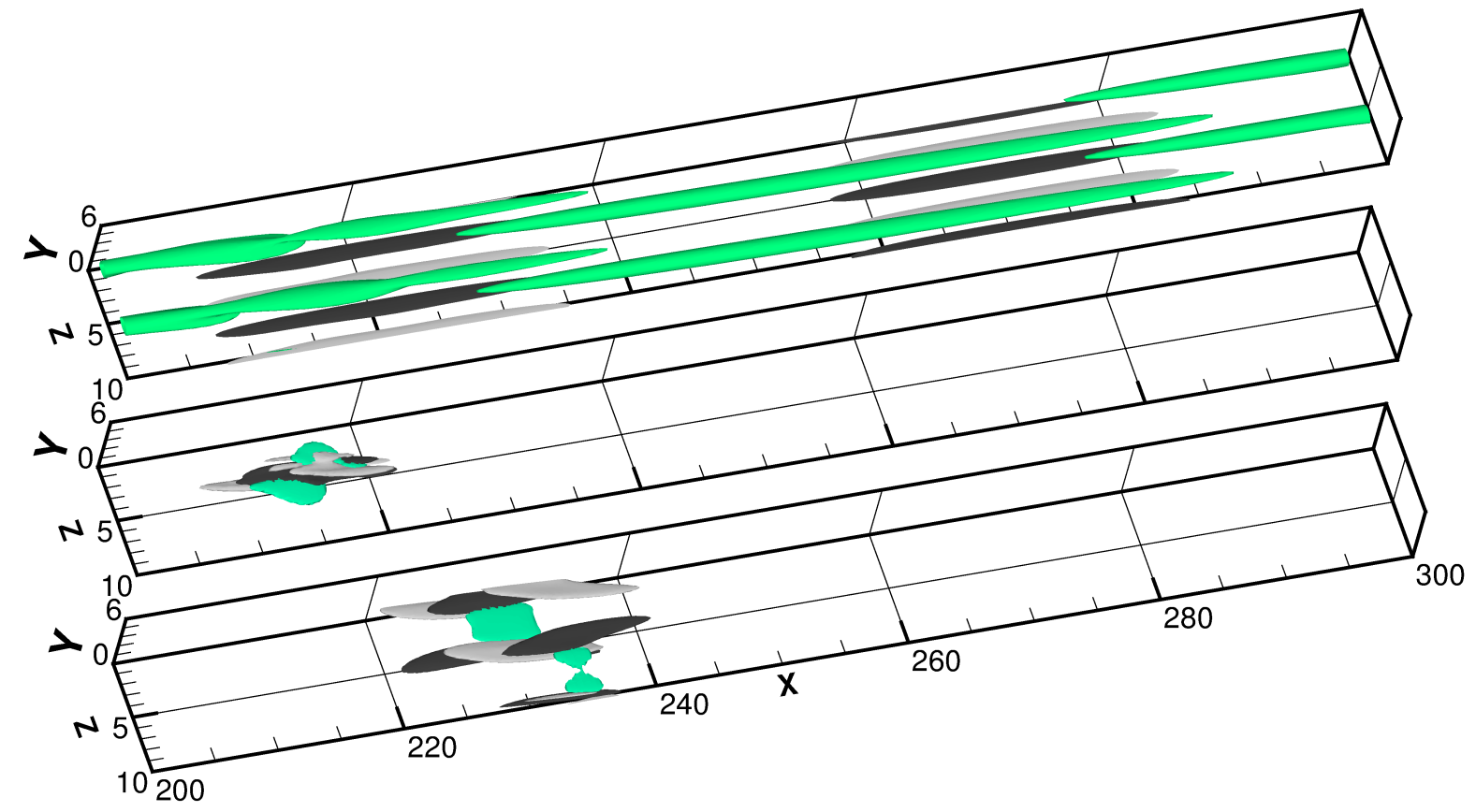

Figure 4: (Color online) Initial perturbations obtained by the non linear optimization for the asymptotic suction boundary-layer at $R e=610$ and target time $T=75$ : iso-surfaces of the optimal perturbations (grey, green online, for the negative streamwise component; dark and light gray for negative and positive streamwise vorticity, respectively) with initial energy $E_{0}=1.2 \times 10^{-7}$ (top frame, surfaces for $u^{\prime}=-0.00017, \omega_{x}^{\prime}= \pm 0.01$ ) and $E_{0}=3.0 \times 10^{-7}$ (middle frame, $u^{\prime}=-0.015$, $\left.\omega_{x}^{\prime}= \pm 0.1\right)$. Initial perturbations obtained by the non linear optimization for the Blasius boundary layer flow at $R e=610$, target time $T=75$, with initial energy $E_{0}=1.2 \times 10^{-7}$ (bottom frame, $\left.u^{\prime}=-0.01, \omega_{x}^{\prime}= \pm 0.06\right)$. Axes are not in the same scale.

The structure of the NLOP found here shows some similarities with that found for the Couette flow (compare with Figure 5 of Ref. [12]) and with that obtained for the BBL flow (see Figure 4, bottom). For all of these shear flows, the NLOP is characterized by streamwise-inclined vortical structures and finite-amplitude patches of streamwise disturbance. However, while for the Couette and the ASBL flow (at least at low Reynolds number) the optimal disturbance does not show any particular symmetry, for the BBL it is symmetrical with respect to a $z=$ const axis. In fact, one can see in Figure 4 (bottom frame), that the NLOP for the Blasius flow at $E_{0}=1.2 \times 10^{-7}$ is composed by a basic structure similar to that of the ASBL, but the disturbance is symmetric with respect to a $z$-aligned axis.

The flow structures can be better analyzed by taking $x$-constant slices of the NLOP, as 


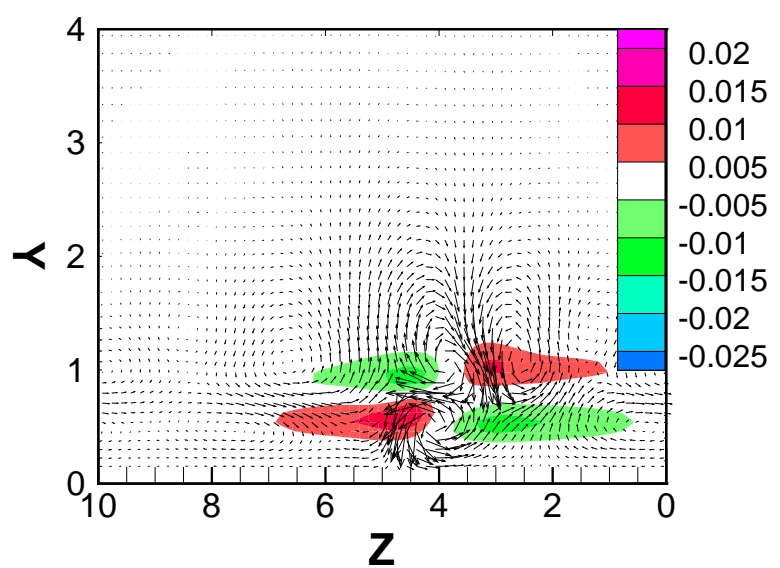

(a)

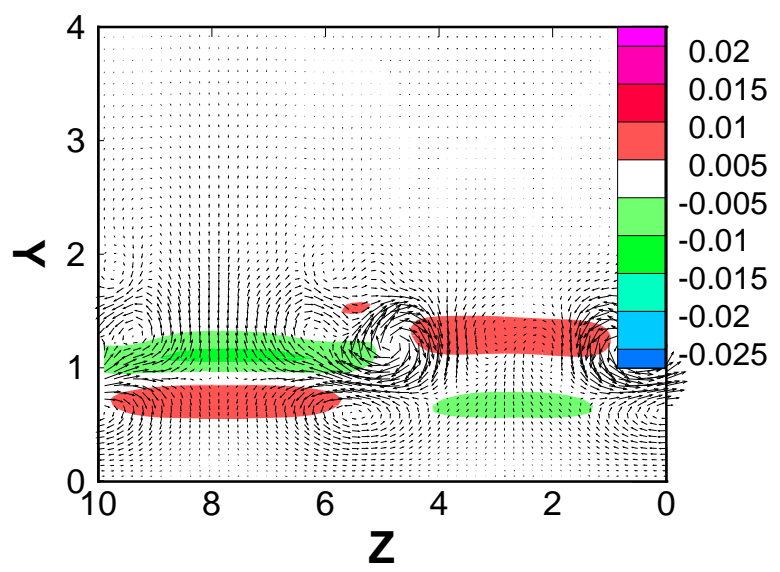

(c)

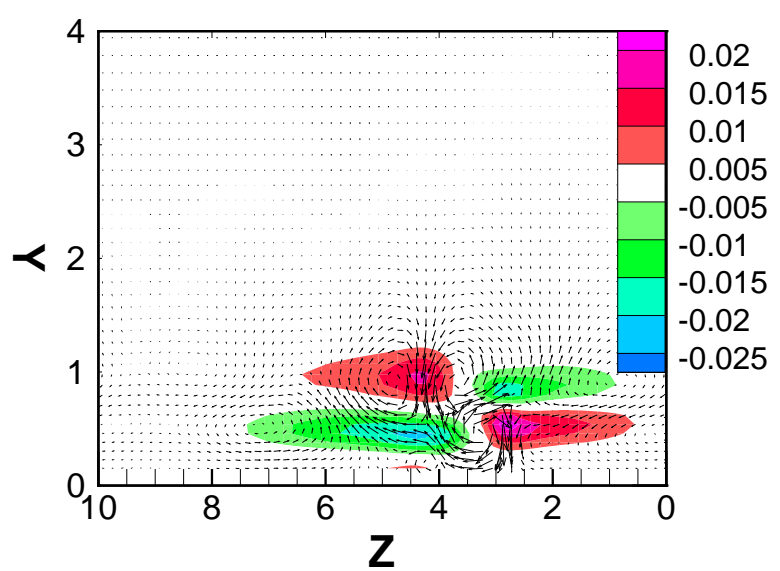

(b)

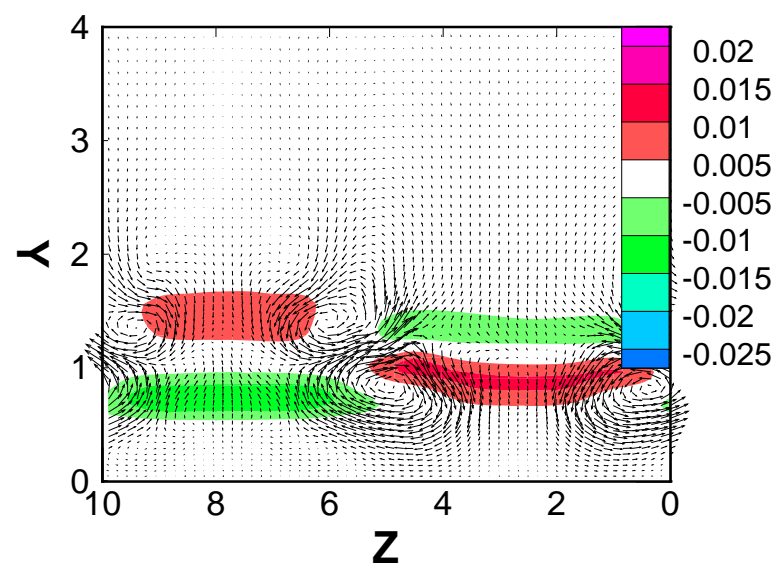

(d)

Figure 5: (Color online) Contours and vectors of the velocity components of the NLOP at initial time obtained with $R e=610, T=75$ for the asymptotic suction boundary layer with $E_{0}=3.0 \times 10^{-7}$, on the planes $x=211$ (a), and $x=215$ (b); for the Blasius boundary layer with $E_{0}=1.2 \times 10^{-7}$, on the planes $x=228(\mathrm{c})$, and $x=232(\mathrm{~d})$. Shaded contours indicate the streamwise disturbance velocity (dark, red online, for positive values; light, green online, for negative ones); vectors represent the wall-normal and the spanwise disturbance velocity components.

provided in the top frames of figure 5. One can observe that the inclined alternated vortices are strictly localized in a narrow zone in the spanwise direction $(2<z<5)$, surrounded by patches of negative (light gray) and positive (dark gray) streamwise perturbation which are alternated in the spanwise and wall-normal direction, at the flanks of the vortices. The differences with respect to the BBL can be analyzed by comparing the top frames (ASBL) 
with the bottom frames (BBL) of figure 5. Concerning the latter, the vortices are localized in two distinct regions in the spanwise direction, connected by alternated patches of streamwise disturbance showing a symmetry in the spanwise direction. This discrepancy is reflected also at target time, as one can notice comparing Figures 6 (a)-(b), for the ASBL, with (c)-(d), for the BBL. For the ASBL, one can observe in figure 6 (a)-(b) the presence of bent streaks along the streamwise vortices, showing strong oscillations in the streamwise and spanwise directions. Whereas, in figures 6 (c)-(d) one can notice that, for the BBL, the perturbations remain spanwise-symmetric, and the vortices as well as the streaks along them are strongly lifted in the wall-normal direction, showing the typical signature of an incipient hairpin vortex.

As proposed in Ref. [40], the disturbance of minimum amplitude capable of triggering turbulence is defined as the minimal seed for a given Reynolds number. Bisecting the value of the initial energy at $T=200$, and checking whether the obtained NLOP is able to induce transition, we have found the energy level of the minimal seed to be about $1.277 \times 10^{-7}$ for $R e=610$. The corresponding maximum amplitudes of the velocity components are $|u|_{\max }=0.029,|v|_{\max }=0.031,|w|_{\max }=0.031$, very close to the values found at lower target time (even if the wall-normal component is now slightly larger than the streamwise one). The minimal seed is sandwiched between the NLOPs shown in figure 7 (a) and (b), for $E_{0}=1.2 \times 10^{-7}$ and $E_{0}=1.35 \times 10^{-7}$, both showing the basic structure provided in figure 4 (middle frame). It is worth to notice that the NLOP keeps the same structure of the minimal seed also for values of the initial energy slightly lower than the minimal seed energy. For larger values of the initial energy, local maxima can be found, as in the case of the Couette flow [12]. Furthermore, for energies larger than the minimal seed one, convergence on the optimum is not assured (see [42]), since transition might be observed at target time. However, since for $T=200$ transition is still not observed for initial energies not much higher than the minimal seed one, we have been able to perform two optimizations with two different initial conditions, in order to check the existence of such local maxima. Thus, we have chosen $E_{0}=2.7 \times 10^{-7}$ (almost double than the energy of the minimal seed), with two different initializations, namely, i) the minimal seed structure, and ii) a symmetrical initial disturbance constructed by mirroring in the spanwise direction the minimal seed structure with respect to the streamwise axis. The energy gain for the first optimization is $E(T) / E_{0}=$ 1641, and the optimal shape is shown in figure 7 (c); whereas, for a symmetrical initial guess, 


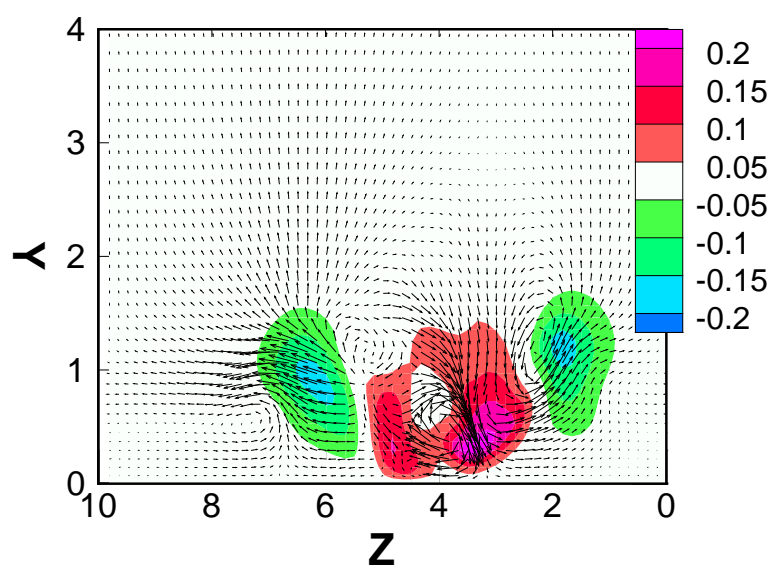

(a)

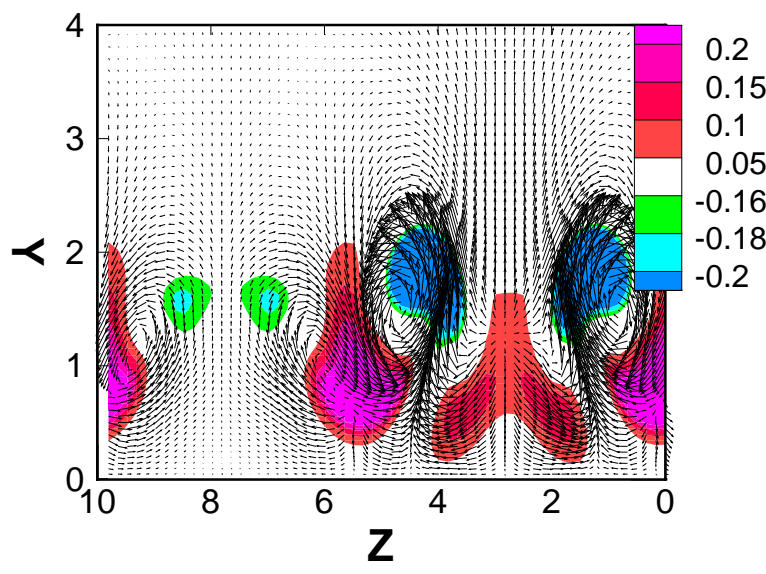

(c)

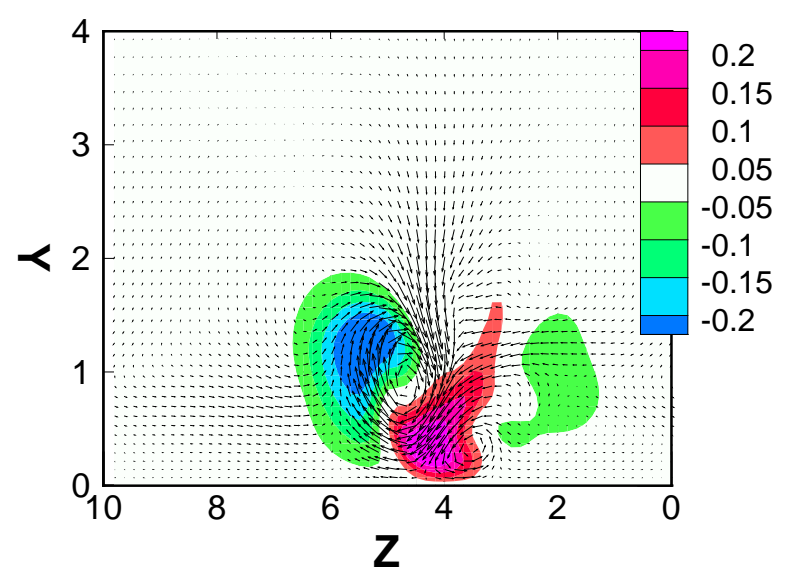

(b)

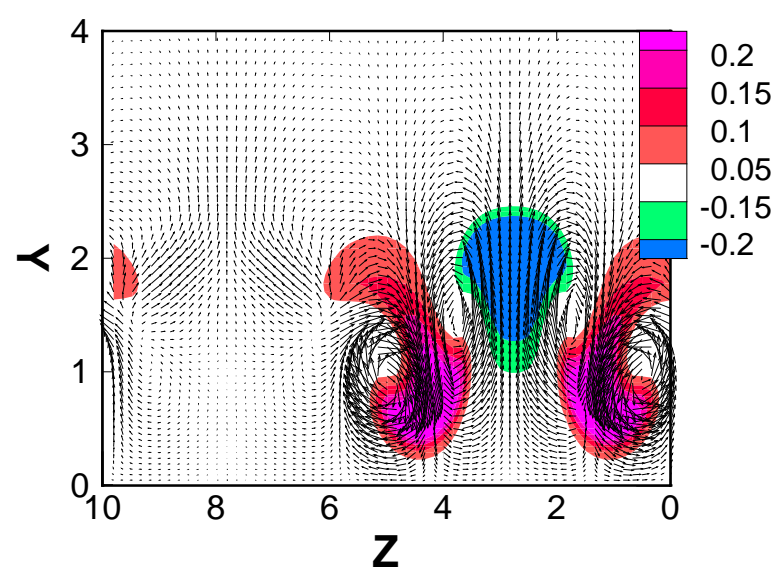

(d)

Figure 6: (Color online) Contours and vectors of the velocity components of the NLOP at target time obtained with $R e=610, T=75$ for the asymptotic suction boundary layer with $E_{0}=3.0 \times 10^{-7}$ on the planes $x=249$ (a), and $x=253(\mathrm{~b})$; for the Blasius boundary layer with $E_{0}=1.2 \times 10^{-7}$ on the planes $x=267$ (c), and $x=273(\mathrm{~d})$. Shaded contours indicate the streamwise disturbance velocity (dark, red online, for positive values; light, green online, for negative ones); vectors represent the wall-normal and the spanwise disturbance velocity components.

the suboptimal structure shown in figure 7 (d) has been found, with energy gain $E(T) / E_{0}=$ 254 (even if the optimization eventually converges to the non symmetrycal solution if the convergence process is continued to lower values of the residual). The comparison between the optimal in figure 7 (c) and the suboptimal in figure 7 (d) proves that replication and spatial spreading of the basic structures is observed for large initial energies, as in the Couette 


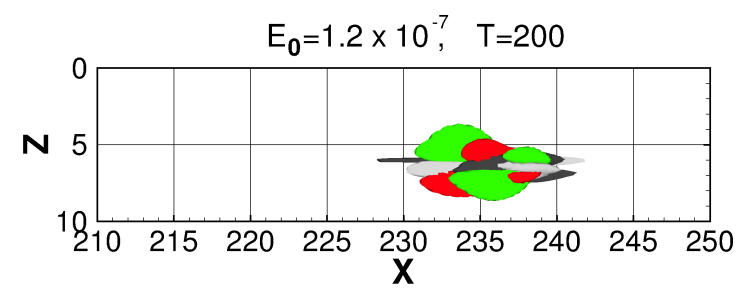

(a)

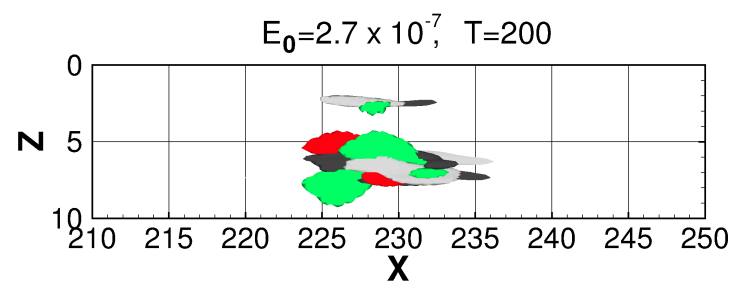

(c)

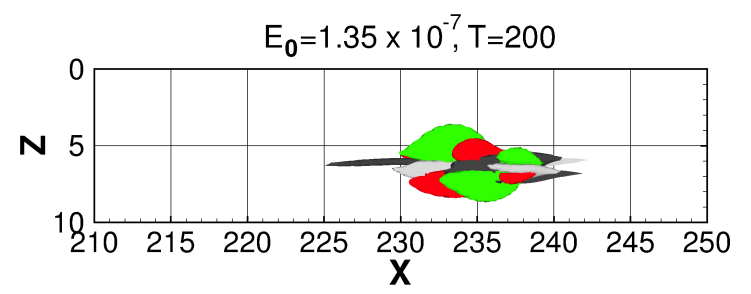

(b)

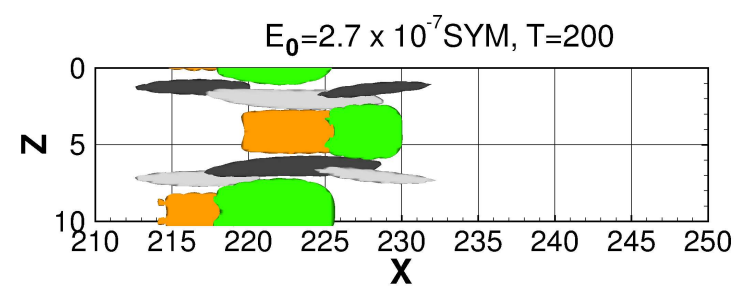

(d)

Figure 7: (Color online) Isosurfaces of the initial perturbations obtained by the non linear optimization for the ASBL at $R e=610$ and target time $T=200$, with initial energies $E_{0}=1.2 \times 10^{-7}$ (a), $E_{0}=1.35 \times 10^{-7}$ (b), $E_{0}=2.7 \times 10^{-7}$ (c) and a suboptimal for $E_{0}=2.7 \times 10^{-7}$ initialized with a symmetric initial perturbation (d). Green and red, for the negative and positive streamwise velocity component; dark and light, for negative and positive streamwise vorticity, respectively, with values $u^{\prime}=-0.005, \omega_{x}^{\prime}= \pm 0.05$ (a-b), $u^{\prime}=-0.01, \omega_{x}^{\prime}= \pm 0.08$ (c), $u^{\prime}=-0.005, \omega_{x}^{\prime}= \pm 0.05$ (d).

flow, but this does not lead to a symmetrisation of the optimal (at least for $R e=610$ ).

The results discussed above show that NLOPs obtained for different shear flows share a similar structure, characterized by inclined vortices along a patch of finite streamwise velocity perturbation (although with a different symmetry). The persistence of this basic structure at different values of the initial energy, target times and for different kind of flows indicates that such a structure, which maximizes the disturbance energy over a finite time, has an intrinsic fundamental importance for shear flows. However, a crucial difference has been found between the basic structure of NLOP found for the ASBL flow (similar to that of the Couette flow) and the BBL flows, namely, the spanwise symmetry of the perturbation. This important difference has motivated the analysis of the structure of the NLOP for several Reynolds numbers provided in the next subsection. 


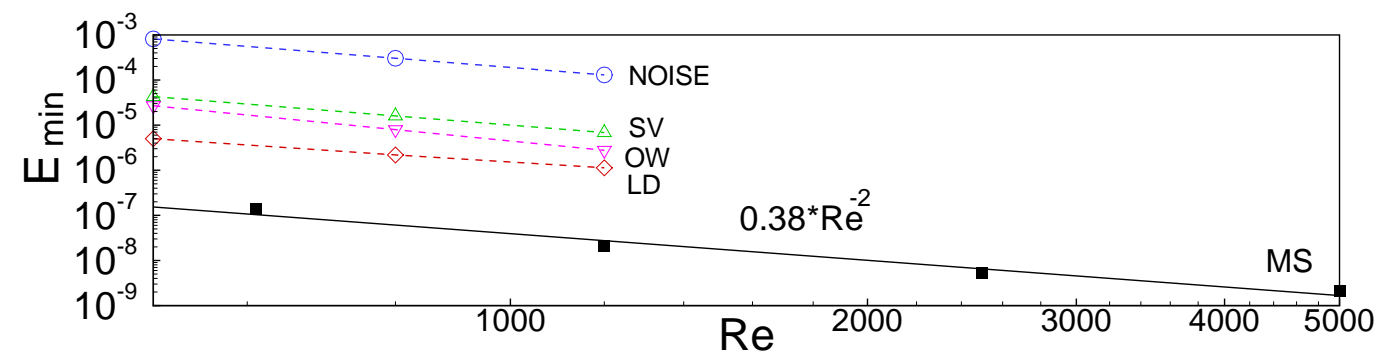

Figure 8: Minimal energy for turbulent transition for the asymptotic suction boundary layer at different Reynolds numbers (solid line). The dashed lines show the minimal energy for different transition scenarios, namely noise (NOISE), streamwise vortices (SV), oblique waves (OW), and localized disturbances (LD), extrapolated from data in [34].
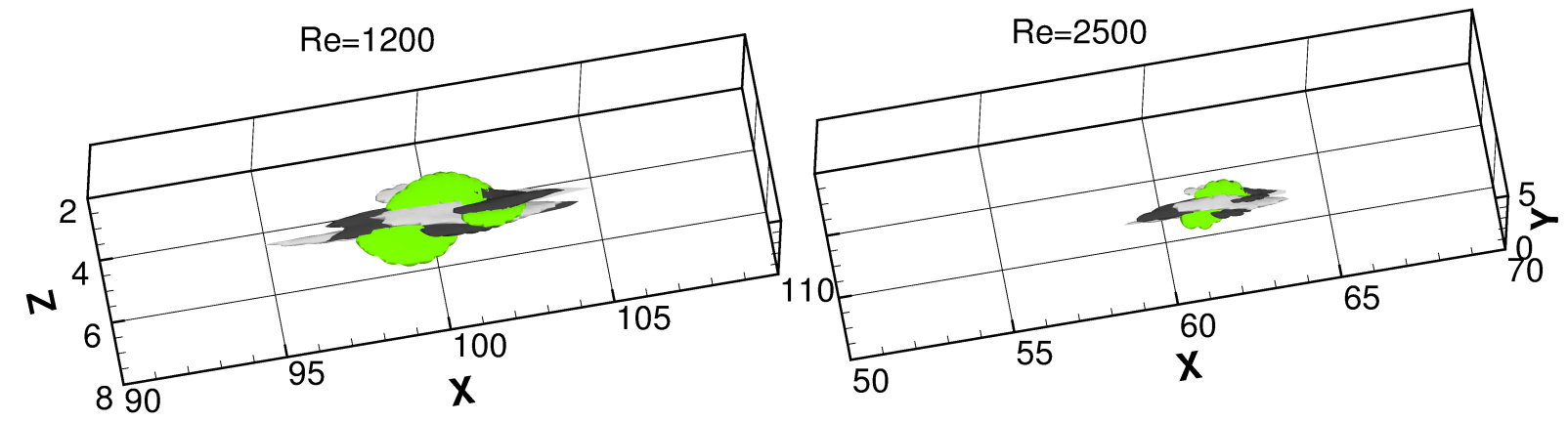

Figure 9: (Color online) Minimal seeds for the asymptotic suction boundary layer for $R e=1200$ (left, with $E_{0}=2.08 \times 10^{-8}$ ) and $R e=2500$ (right, with $E_{0}=4.16 \times 10^{-9}$ ) with target time $T=150$ : iso-surfaces of the negative streamwise component, $u^{\prime}=-0.0025$ (green) and of the negative and positive streamwise vorticity, $\omega_{x}^{\prime}=-0.045$ (black and white, respectively)

\section{B. Reynolds number analysis}

The laminar profile of the ASBL given by equation (2) is linearly stable for $R e<54382$ [23], which is about 100 times the critical Reynolds for a Blasius boundary layer [44]. Thus, the value of $R e=610$ employed in the previous analysis is largely subcritical for the ASBL. Therefore, to generalize our results, we have extended the analysis to larger Reynolds numbers, namely, $R e=1200,2500,5000,10000$. For each value of the Reynolds number but the latter, the value of the initial energy has been bisected in order to approximate the 

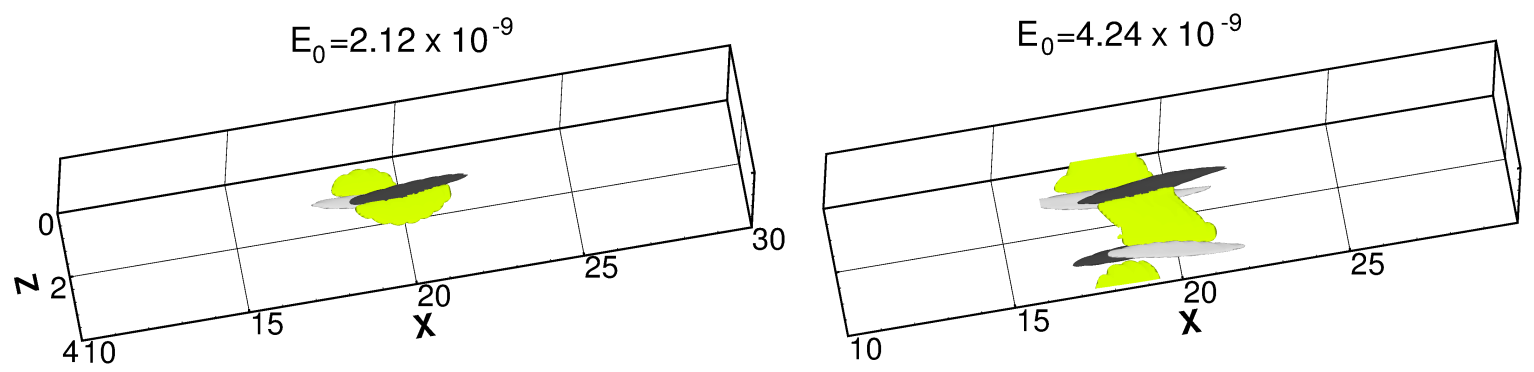

Figure 10: (Color online) Non linear optimal perturbations for the asymptotic suction boundary layer at $R e=5000$ for $E_{0}=2.12 \times 10^{-9}$ (left) and $E_{0}=4.24 \times 10^{-9}$ (right) with target time $T=100$ : iso-surfaces of the negative streamwise component, $u^{\prime}=-0.001$ (green) and of the negative and positive streamwise vorticity, $\omega_{x}^{\prime}= \pm 0.045$ (black and white, respectively)

minimal seed with a two-digit accuracy. The solid line in Figure 8 shows the energy of the minimal seed, $E_{m i n}$, versus the Reynolds number, for $R e=610,1200,2500,5000$. Whereas, the dashed lines in the figure reproduce the results of Ref. [33, 34], for four different initial perturbation structures: i) random three-dimensional noise (NOISE); ii) streamwise vortices (SV), obtained by a local spatial optimization; iii) spatially extended oblique waves (OW), obtained by a local spatial optimization; iv) localized disturbances (LD) consisting of two alternated counter-rotating pairs of streamwise vortices. One can notice that the transition threshold provided by the minimal seed energy is amost two orders of magnitude lower than the energy thresholds found for spatially extended disturbances such as the streamwise vortices and the oblique waves. Moreover, $E_{\min }$ is one order of magnitude lower than the minimal energy found for the localized perturbations selected in Ref. [34]. We can also observe in figure 8 that $E_{\text {min }}$ varies with $R e$ roughly following a power law $R e^{-2}$, whereas Levin et al. [33] found a -2.1 exponent for the SV and NOISE perturbations and $\mathrm{a}-2.8$ exponent for the $\mathrm{OW}$ one. It is worth to notice that in Ref. [16], computing minimal seeds, a power law $R e^{-2.7}$ has been found for a Couette flow in a small domain (whereas the OW scenario was characterized by a -2 exponent for the same configuration). Concerning the velocity amplitudes, for $R e=1200$ the minimal seed is characterized by $|u|_{\max }=0.014,|v|_{\max }=0.015,|w|_{\max }=0.017$. Whereas the minimal LD triggering transition in Ref. [34] was characterized by $|v|_{\max }=0.0124$, very close to the minimal amplitudes found here, but $|u|_{\text {max }}=0.0$. Thus, the large difference in the transition thresholds can 


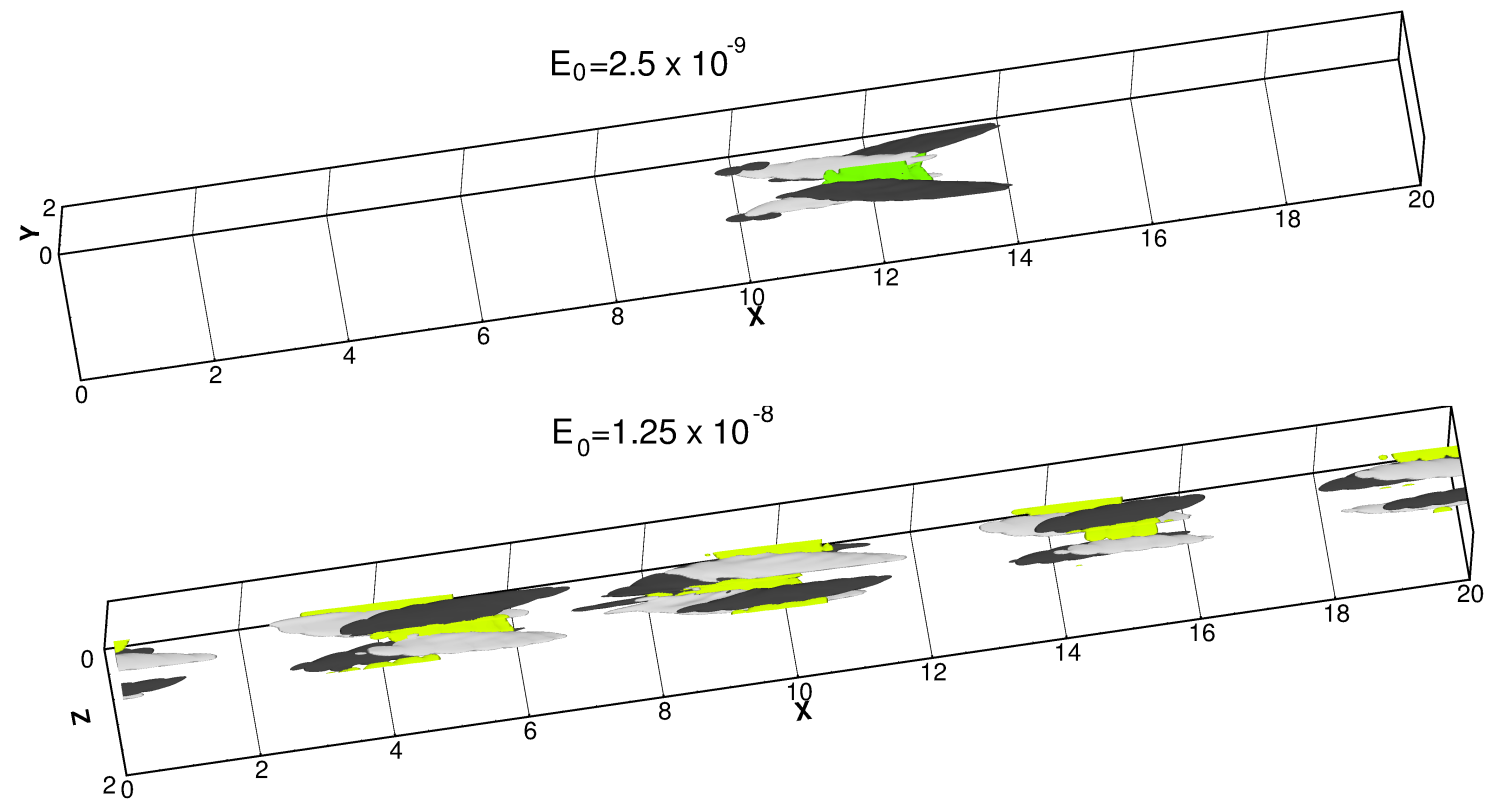

Figure 11: (Color online) Non linear optimal perturbations for the asymptotic suction boundary layer at $R e=10000$ with $E_{0}=2.5 \times 10^{-9}$ (top), and with $E_{0}=1.25 \times 10^{-8}$ (bottom) with target time $T=50$ : iso-surfaces of the negative streamwise component, $u^{\prime}=-0.002$ (green) and of the negative and positive streamwise vorticity, $\omega^{\prime}{ }_{x}= \pm 0.045$ (black and white, respectively)

be linked on the complete absence of streamwise velocity disturbances in Ref. [34], which appears to be a crucial feature for inducing a rapid transition to turbulence using low-energy perturbations. Two other crucial elements which might explain the difference between the energy thresholds for the LD and the minimal seed are: i) the larger spatial extension of the LDs, which makes them more energetic than the minimal seed for similar associated amplitudes; ii) the fact that the vortices are perfectly aligned with the streamwise axis, and spanwise-symmetric with respect to this axis, whereas the perturbations inducing the largest growth by non linear mechanisms appear to be characterized by a finite inclination with respect to the streamwise axis, and do not show any spanwise symmetry. Thus, it appears that a non linear optimization is crucial to determine the order of magnitude of the minimal thresholds for transition to turbulence, and for accurately determining the shape and typical length scales of the minimal perturbation capable of inducing transition to turbulence.

As shown in Figure 9, the shapes of the minimal seed for $R e=1200$ and $R e=2500$ are very similar to the ones described in the previous section for $R e=610$. They are composed 


\begin{tabular}{llllll}
\hline$R e=$ & 610 & 1200 & 2500 & 5000 & 10000 \\
$l_{x}=$ & 18.2 & 8.6 & 4.1 & 2.9 & 1.7 \\
$l_{z}=$ & 8.6 & 4.4 & 2.7 & 1.94 & 1.3 \\
\hline
\end{tabular}

Table III: Streamwise and spanwise dimensions, $l_{x}, l_{z}$ of the minimal seed for the considered Reynolds numbers, measured as the largest spanwise and streamwise lengths of the flow regions where $\left|u^{\prime}\right|>0.001$

by streamwise alternated positive and negative vortices with a finite inclination with respect to the streamwise and wall-normal axis. It is worth to notice that, even if the basic structure is the same, the minimal seed is much more localized for higher values of the Reynolds number, as shown in table III. In particular, the streamwise and spanwise lengths appear to be almost halved for an increase of $R e$ of a factor two.

For a fluid with a given kinematic viscosity, the dependence on $R e$ of the typical length scales of the wall-structures, such as the streaks, is more complex for the ASBL than for the BBL. For the ASBL the Reynolds number can be varied either by changing the freestream velocity $U_{\infty}$ (keeping the suction velocity fixed), or by changing the suction velocity, thus modifying the displacement thickness $\delta^{*}$ (but keeping $U_{\infty}$ fixed). Yoshioka et al. [54] have experimentally measured the typical length scales of wall structures induced by free streamturbulence in the ASBL, concluding that: i) if $V_{S}$ and $\delta^{*}$ are kept constant, the spanwise spacing of the streaks varies with $U_{\infty}^{-1}$; ii) if $U_{\infty}$ is kept constant and $\delta^{*}$ is changed, the dimensional spacing of the streaks remains constant. In the present work, we change the Reynolds number by keeping $U_{\infty}$ constant, modifying the suction velocity $V_{S}$; therefore, we are increasing the reference length, $\delta^{*}$, when the Reynolds number increases. Thus, a decrease of a factor 2 of the minimal seed size corresponding to a twofold increase of the reference length $\delta^{*}$ is consistent with the results in Ref. [54], since the dimensional typical length scales of the streaks will not change with $R e$ when $U_{\infty}$ is kept constant. We can thus conclude that, at least for Reynolds numbers in the range $[610,10000]$, the typical length scales of the NLOPs change accordingly to the streaks spacing measured in Ref. [54]. This explains why we have chosen to use smaller domain lengths for larger Re.

Another important feature of the minimal seed shown in figure 9 for $R e=1200$ and 2500 is that, as for $R e=610$, it does not present any symmetry in the spanwise direction. How- 
ever, when the Reynolds number is increased to $R e=5000$, two types of non linear optimal solutions have been found. Figure 10 shows the result of the non linear optimization for two initial energies, $E_{0}=2 \times 10^{-9}$ and $E_{0}=4 \times 10^{-9}$, for $T=100$. One can observe that the latter is almost symmetrical, roughly corresponding to a mirroring of the former with respect to the streamwise axis. The minimal seed for this Reynolds number is sandwhiched between these two solutions with different symmetries. Further bisections between these two values indicate that the minimal seed is not symmetrical. However, the presence of a symmetrical non linear optimal is an indication of the change in the optimal dynamics that is observed at larger Reynolds numbers. In fact, for $R e=10000$, only symmetrical optimal disturbances have been obtained. Figure 11 shows two of them for two energy levels and a short target time $T=50$. This result has been verified by performing optimizations at a larger, supercritical Reynolds number, $R e=65000$, for which a symmetrical minimal seed has been found as well, preserving the same structure. This indicates that a value of the Reynolds exists between 5000 and 10000 for which the NLOP changes from a nonsymmetric shape to a symmetric one. Therefore, for sufficiently high Reynolds numbers, the structure of the minimal seed becomes similar to the one of the BBL. This behavior can be explained considering that we are changing the Reynolds number $R e=U_{\infty} / V_{S}$ by modifying the suction velocity $V_{s}$; therefore, for high values of Re, the influence of $V_{S}$ tends to become smaller and the velocity profile tends to be closer to that of the BBL flow. At some "critical" Reynolds number, the modification of the ASBL profile towards a profile closer to the BBL one induces a change in the symmetry of the NLOP.

In the next section we will compare the route to turbulence at low Reynolds number for the $\mathrm{ASBL}$ and the BBL, in order to understand which differences in the base flow profile can induce different symmetries on the non linear optimal solutions, and link these results to the change of symmetry observed at high Reynolds numbers in the ASBL

\section{The route of the non linear optimal perturbations to turbulence}

In this section, we analyze by DNS the evolution towards turbulence of the NLOP obtained for the ASBL with $R e=610$ and $T=75$, providing a comparison with the NLOP of the BBL for the same conditions [10]. In order to achieve transition, the two perturbations have different energy, namely, $E_{0}=3.0 \times 10^{-7}$ for the ASBL and $E_{0}=1.2 \times 10^{-7}$ for the 

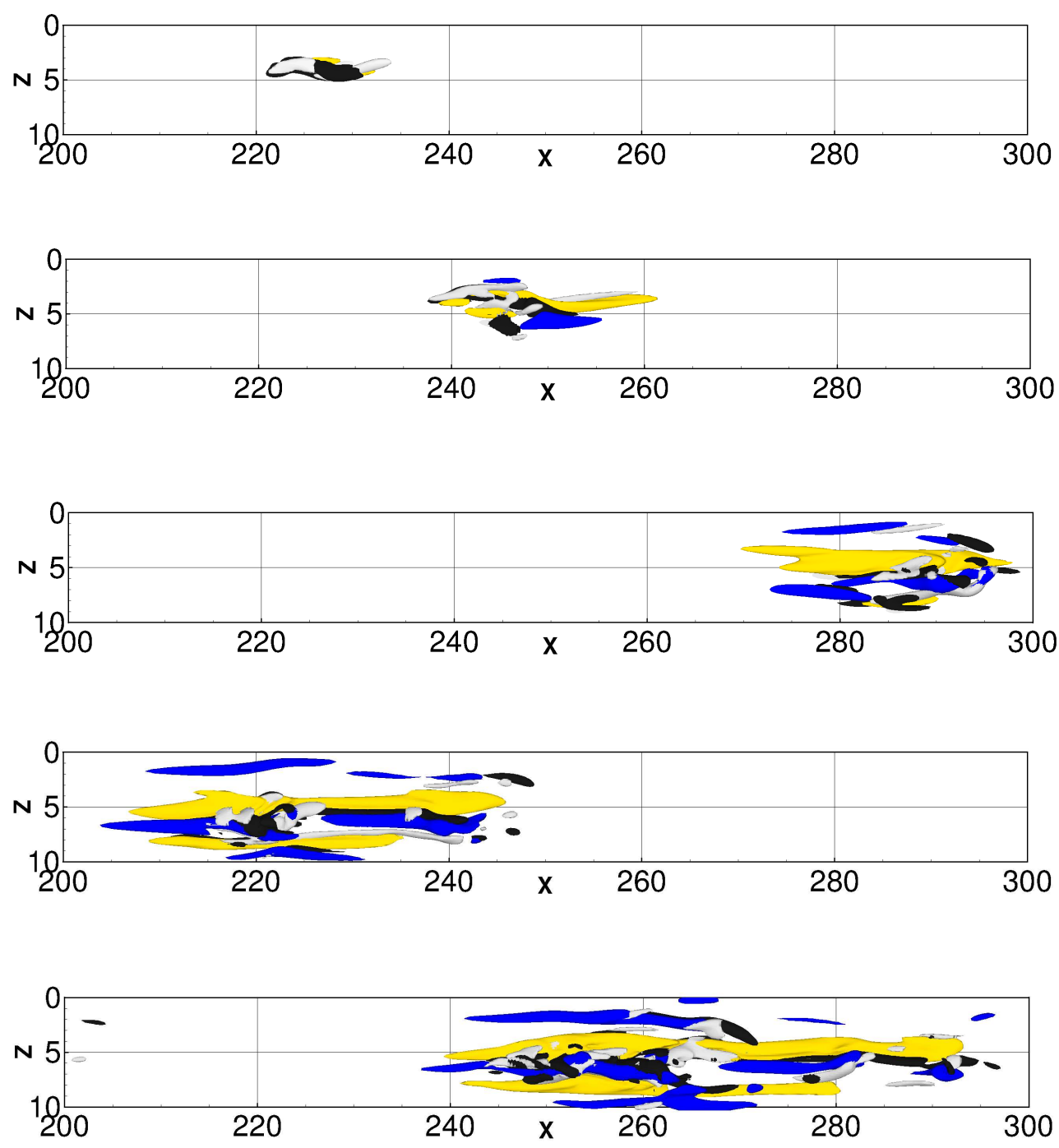

Figure 12: (Color online) Snapshots of the evolution in time of the selected NLOP for the ASBL: iso-surfaces of the streamwise velocity and vorticity perturbations (yellow and blue, for $u^{\prime}= \pm 0.15$, respectively; black and white, $\omega^{\prime}{ }_{x}= \pm 0.2$, respectively) at $t=40,80,140,200,260$ (from top to bottom).

BBL. In the case of the BBL, being the flow non-parallel, we do not impose periodicity in the streamwise direction. Thus, we have used a domain two times longer in $x$ than that used for the ASBL, in order to follow the evolution of the perturbation for a sufficiently long time before the disturbance leaves the domain. The number of grid points in the streamwise direction has been doubled as well, in order to maintain the same local grid resolution. 

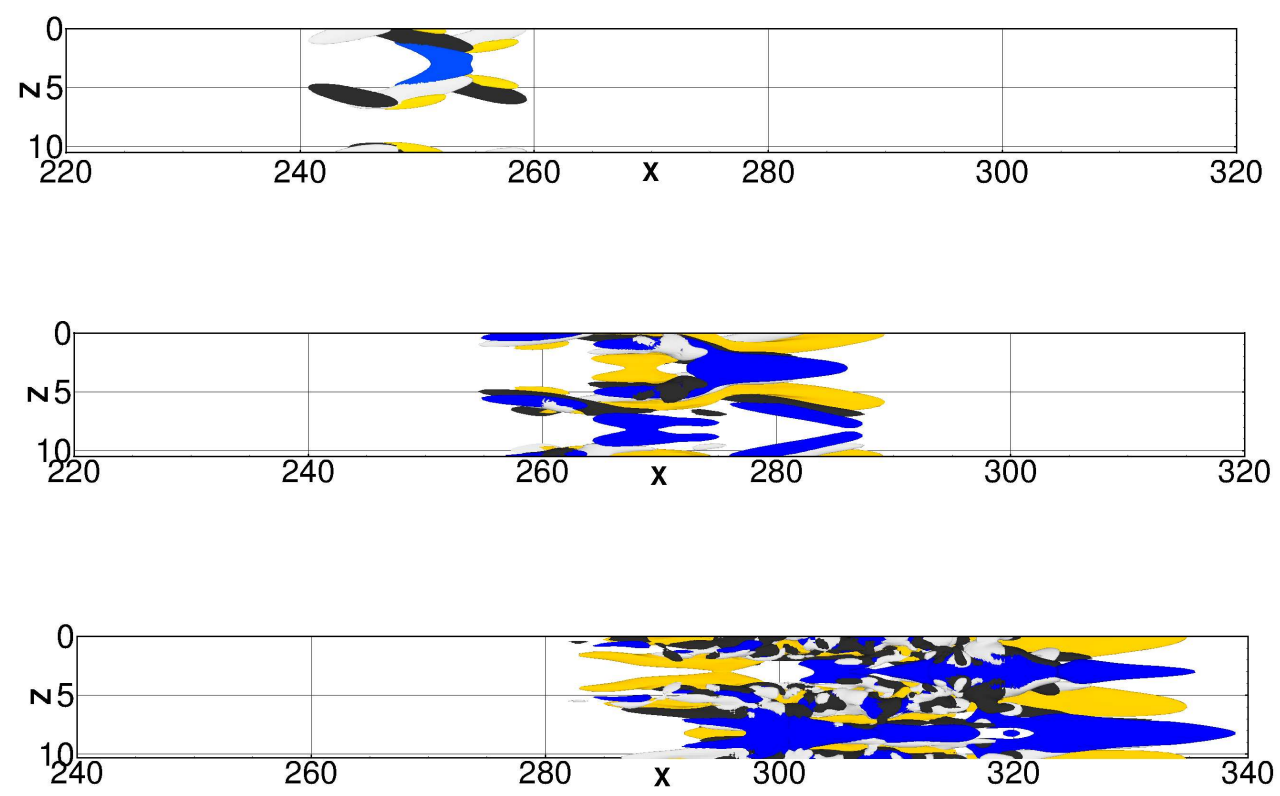

Figure 13: (Color online) Snapshots of the evolution in time of the selected NLOP for the BBL: iso-surfaces of the streamwise velocity and vorticity perturbations (yellow and blue, for $u^{\prime}= \pm 0.1$, respectively; black and white, $\omega_{x}^{\prime}= \pm 0.2$, respectively) at $t=40,80,140$ (from top to bottom).
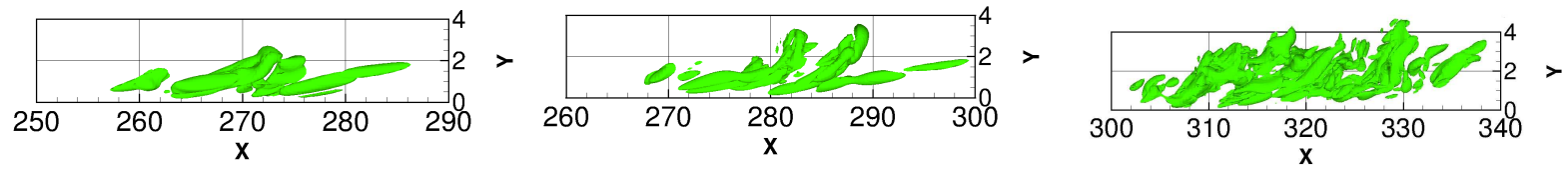

Figure 14: (Color online) Snapshots of the evolution in time of the selected NLOP for the BBL: isosurfaces of the Q-criterion for $t=80,100,160$ (from left to right) .
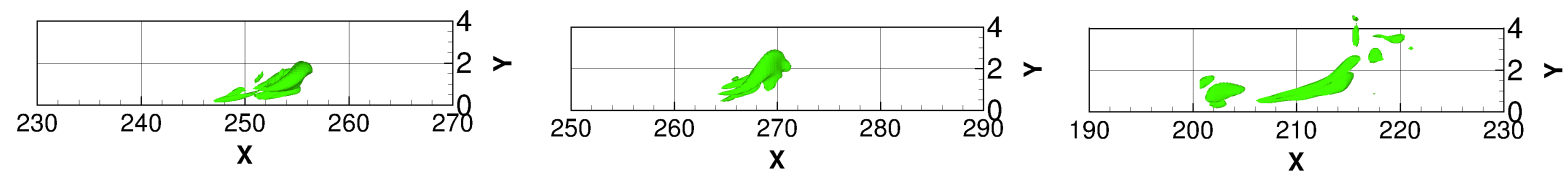

Figure 15: (Color online) Snapshots of the evolution in time of the selected NLOP for the ASBL: isosurfaces of the Q-criterion for $t=80,100,160$ (from left to right) . 
A qualitative picture of the transition process initiated by the NLOP for the ASBL is given in figure 12, showing the streamwise vorticity (black and white surfaces) and velocity (blue and yellow) perturbations. At $t=40$ (first frame), the initial vortices increase their strength and streamwise inclination. This first phase is similar to that found for the BBL, and appears to depend on the action of non linear coupling terms linked to the components of the streamwise vorticity, such as $w w_{z}$, as explained in detail in Ref. [10]. At the same time, the streamwise velocity perturbation increases its amplitude, due to a modified lift-up effect [10]. In fact, since the initial vortices are inclined, the generated streaks are modulated in the streamwise direction, as shown in the second frame for $t=80$. In particular, a main high-speed bent streak (yellow) is created, flanked by two weaker low-speed ones. On such streaks, localized patches of vorticity are observed (see the third frame for $t=140$ ), which are originated from the splitting of the initial inclined vortices. The bent streaks continue to be fed by the streamwise vortices, elongating in the streamwise direction, as shown in the fourth frame for $t=200$. However, in the regions of larger vorticity, stronger modulations of the streaks are induced, leading the wave packet to break-up starting from a localized region $(x=250$ in the fifth frame for $t=260)$. Such a scenario recalls the mechanism of secondary instability of streamwise streaks which triggers bypass transition in boundary-layer flows [4, 47]. In particular, since the initial disturbance is not symmetric, the streaks are characterized by sinuous oscillations, which represent the primary instabilities of streamwise streaks [3, 4]. However, in the non linear optimal case, this mechanism is much more rapid than the one relying on the linear growth of streamwise-aligned streaks and successive saturation and secondary instability. In fact, the initial inclined vortices can create bent streaks in a short time, leading to break-up without experiencing secondary instability, due to their spanwise modulations [51]. The whole transition process recalls the first phases of the disturbance evolution on the periodic orbit recently found by bisection in a small domain (see Ref. [31]). However, since the NLOP considered here does not lay on the edge of chaos as the mentioned periodic orbit, transition to turbulence is reached after the bursting phase.

Despite the similarity of the initial optimal disturbances, the non linear route to transition described here shows important differences with respect to that found in the non-parallel case. In fact, for the BBL case, the perturbation maintains the initial symmetry of the 
NLOP up to large times (obviously, before turbulence is initiated). As shown in figure 13, the initial symmetric inclined vortices transport the flow momentum causing an amplification of the streamwise component of velocity along them and inducing the creation of low- and high-momentum zones showing a $\Lambda$ and an $X$ shape, respectively (see the blue and yellow surfaces in the first frame for $t=40$ ). This $\Lambda$ structure of the perturbation is maintained at larger time (see the second frame for $t=80$ ), and the symmetric inclined vortices connect their fronts to create a $\Lambda$-vortex, which eventually turns into a hairpin vortex which leads the flow to break-up (third frame for $t=140$ ). The formation of the hairpin for the BBL can be clearly seen in figure 14 at times $t=80$ (a), $t=100$, (b), and $t=160$, where the green surfaces show the Q-criterion, highlighting the regions of high vorticity. At $t=80$, one can observe that the initial vortices, tilted by the mean flow, are inclined at a small angle with respect to the streamwise axis $\left(\approx 7^{\circ}\right)$. However, at larger times, the vortices in the heart of the wave packet increase their inclination, reaching angles of about $35^{\circ}$. This phase coincides with the formation of the hairpin vortex (two of them are visible in the second frame at $t=100$ ) which grows in size and splits into smaller hairpin vortices, leading very quickly to a turbulent spot (see the third frame at $t=160$ ). On the other hand, for the ASBL, although the initial vortices show a similar wall-normal inclination with respect to the streamwise direction, as shown in the first and second frame of figure 15, the head of the hairpin vortex cannot be created due to the lack of symmetry of the perturbation. Thus, the vorticity does not spread in space as in the BBL case, but remains localized in a narrow region in the streamwise direction (see the third frame at $t=160$ ).

The differences between the transition paths in the ASBL and BBL case can be analyzed by extracting the rms values of the three components of the velocity perturbation, as shown in figure 16, the thick lines referring to the ASBL, the thin ones to the BBL. In the BBL flow, the three components of velocity grow more rapidly and achieve larger rms values than in the ASBL case (see figure 16 (a)). Concerning the vorticity perturbation, shown in figure 16 (b), in the BBL case all of the three components grow more rapidly; the largest differences between the two flows are recovered for the wall-normal and spanwise vorticity, which attains values almost one order of magnitude larger than in the ASBL case. This can be explained by observing that the vorticity components $\omega_{z}^{\prime}$ and $\omega_{y}^{\prime}$ have large values at the head and legs of the hairpin which characterize the BBL route to transition. In fact, plotting the $\omega^{\prime}{ }_{z}$ and $\omega^{\prime}{ }_{y}$ surfaces for the parallel and non-parallel flow cases at $t=100$, as 


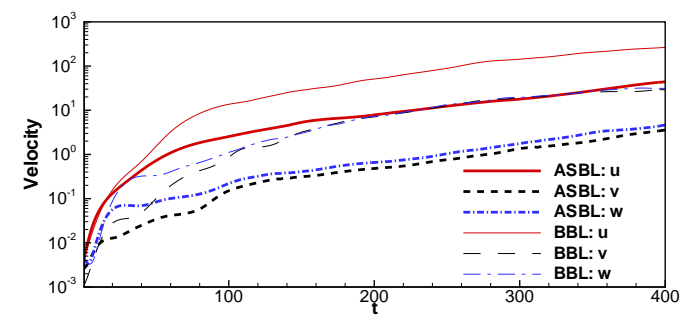

(a)

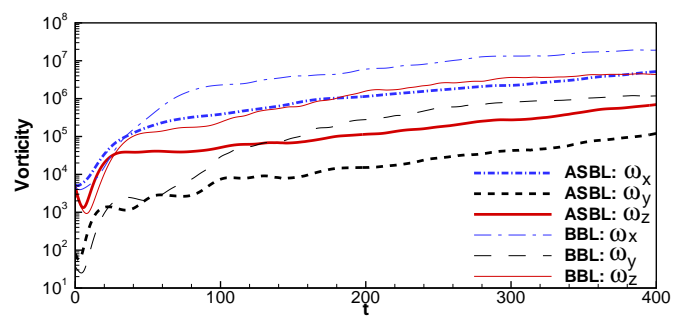

(b)

Figure 16: (Color online) Evolution in time of the rms values of the three components of velocity (solid lines for $u^{\prime}$, dashed for $v^{\prime}$, dashed-dotted for $w^{\prime}$ ) (a) and vorticity (solid lines for $\omega_{z}^{\prime}$, dashed for $\omega^{\prime}$, dashed-dotted for $\omega_{x}^{\prime}$ ) (b) for a DNS initialized by the selected NLOP for the ASBL (thick lines) and the BBL (thin lines).
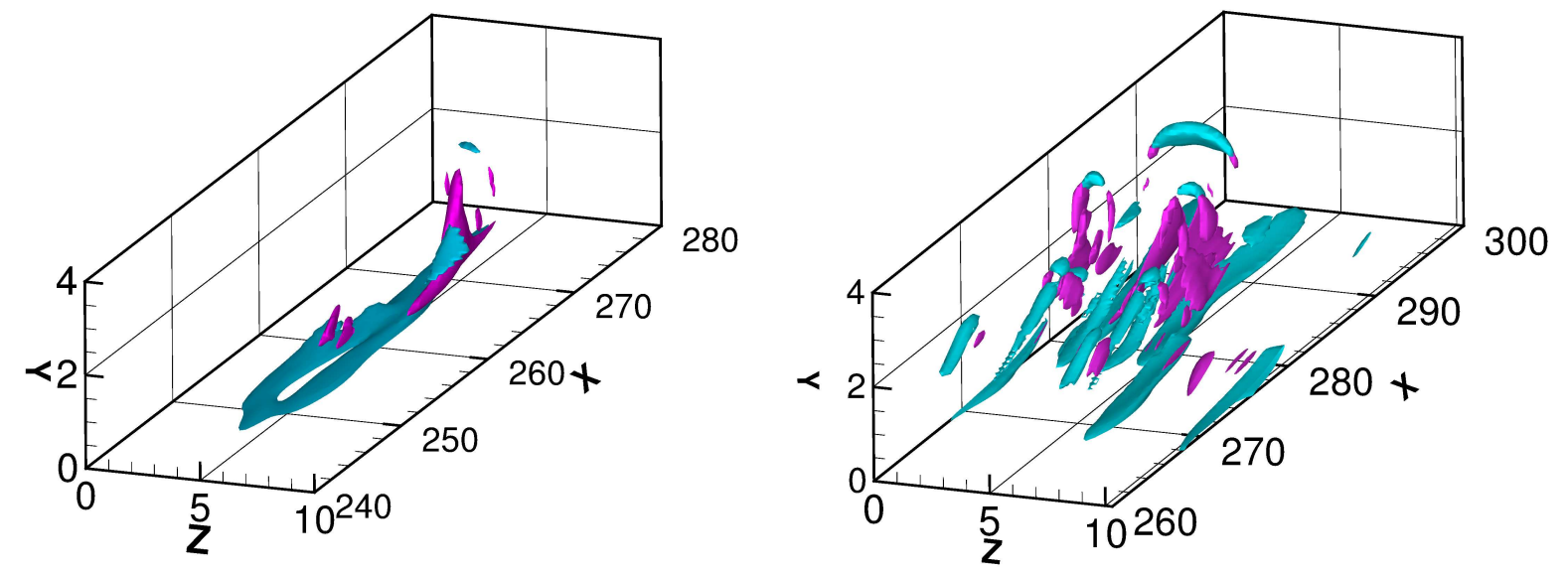

Figure 17: (Color online) Snapshots of the evolution in time of the selected NLOP for the ASBL (left frame) and the BBL (right frame): spanwise and wall-normal vorticity (blue surfaces for $\omega^{\prime}{ }_{z}=0.8$, red ones for $\omega^{\prime}{ }_{y}=0.65$.

provided in figure 17 (a) and (b), respectively, one can observe that these two components of the vorticity perturbations are much more extended in space, and larger in magnitude in the non-parallel case than in the parallel one (both components are about 30\%). In particular, the vorticity surfaces are localized at the head and legs of the hairpin vortices, explaining the larger growth of such terms with respect to the streamwise vorticity.

In order to better understand the differences between the two optimal transition paths, it is worth to analyze the evolution of the symmetric optimal perturbation superposed to the 

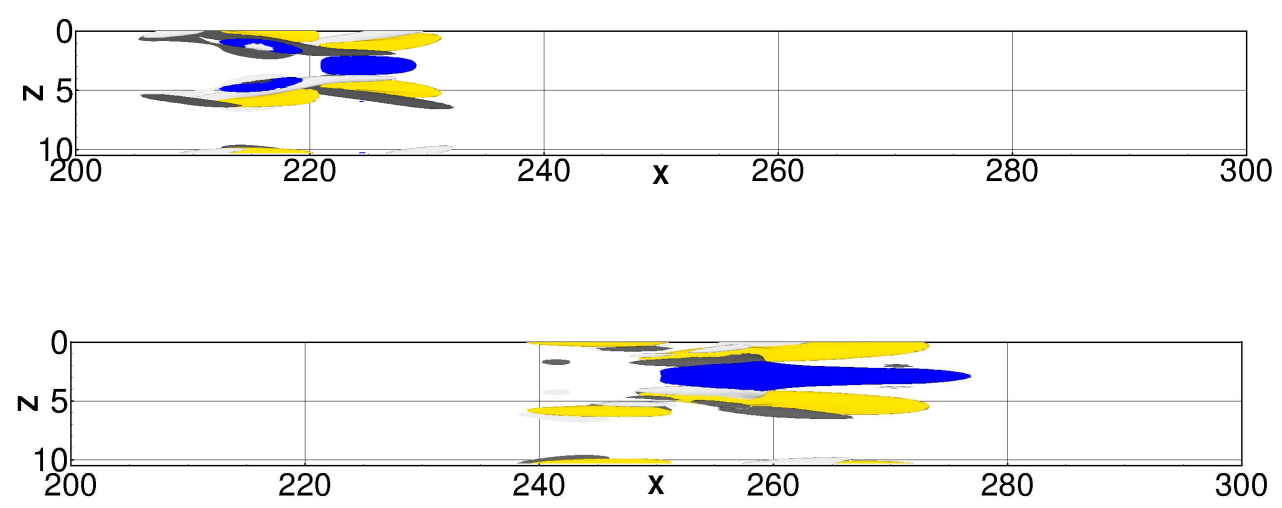

Figure 18: (Color online) Snapshots of the evolution in time of the selected NLOP obtained for the BBL, injected in the ASBL flow: iso-surfaces of the streamwise velocity and vorticity perturbations (yellow and blue, for $u^{\prime}= \pm 0.1$, respectively; black and white, $\omega_{x}^{\prime}= \pm 0.2$, respectively) at $t=$ 80,140 (from top to bottom).
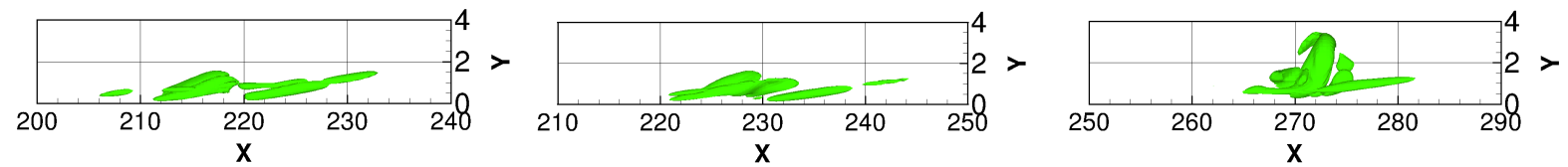

Figure 19: (Color online) Snapshots of the evolution in time of the selected NLOP obtained for the BBL, injected in the ASBL flow: isosurfaces of the Q-criterion for $t=80,100,160$ (from left to right).

ASBL base flow at low Reynolds number. Thus, we inject the selected NLOP obtained for the BBL in the ASBL flow, and analyze its evolution in time. Figure 18 shows the streamwise vorticity (black and white) and velocity (yellow and blue) perturbations at $t=80$ and $t=140$. Comparing with figures 13 (b) and (c), one can observe that the vortices as well as the low- and high-momentum regions are straighten up and lose part of their inclination. As a result, at $t=140$ one can observe three alternated streaks with weak vortices on their flanks. In particular, even if the initial perturbation is symmetric with respect to a $z=$ const axis, the hairpin vortex is not formed at $t=100$. This is clearly shown in figure 19, providing the Q-criterion isosurfaces. One can see that at $t=80$ (a) and at $t=100$ (b) the inclination of the vortices is weak and begins to grow only at $t=160$, turning eventually in a hairpin 
structure at $t \approx 200$. This can be also inferred by analyzing in figure 20 the time evolution of the rms value of the three velocity and vorticity components, and comparing them with the BBL case. As provided by the thick lines in figure 20 (a), the initial growth of the velocity components of the NLOP injected into the ASBL is initially similar to the one characterizing the BBL. However, at $t \approx 40$, the spanwise velocity component begins to decrease, and the wall-normal and the streamwise ones strongly decrease their growth rate. Concerning the vorticity components, at approximately the same time they all begin to decrease, the $\omega_{z}^{\prime}$ and the $\omega_{y}^{\prime}$ with a larger rate than the $\omega_{x}^{\prime}$. This decrease is due to the absence of the hairpin vortex at $t \approx 100$; in fact, it begins to grow only at $t \approx 200$, when the growth of all of the vorticity components is observed. Thus, we can say that the mechanism of formation of the hairpin, which is responsible for the strong growth of all of the vorticity components in the BBL case, is delayed for the ASBL, making a "sinuous" instability mechanism more effective in inducing rapidly transition, like in the case of the Couette flow [12]. Thus, in the case of the ASBL a symmetrical optimal perturbation is less effective in inducing transition than a non-symmetrical one. As observed in figure 15, such a delay appears to be due to the lower wall-normal inclination of the vortices with respect to the streamwise axis. This can be linked to a simple mechanism of transport of the perturbation by the mean flow. In fact, injecting the same initial vortical structure in the ASBL and the BBL flows, in the latter case the highest part of the vortex will experience a lower streamwise base velocity as it is advected downstream, so it will decelerate with respect to its lowest part. This will induce an increase of the inclination of the initial vortex in the wall-normal direction, leading to a connection of the fronts of the two vortices, finally creating an hairpin structure. This is clearly shown in figure 21, providing the base flow vectors at $R e=610$ for the ASBL (left frame) and the BBL (right frame) and the effect they have on the same vortical structure evolving in time up to $t=100$. For the ASBL flow, the profile is fuller than the BBL one, so the mechanism of inclination by the mean flow is weaker, delaying the formation of the hairpin vortex from an initial symmetric perturbation. Therefore, the zones of strong velocity deficit (in blue in the figure) remain closer to the wall, delaying the formation of inflection points in the mean flow profile, and the consequent transition to turbulence. Moreover, we have verified that this effect is not due to the parallelism of the ASBL flow. In fact, a non-linear optimization performed for a parallel flow having the same Blasius profile with $R e=610$ at all abscissae, gives a symmetric NLOP. 


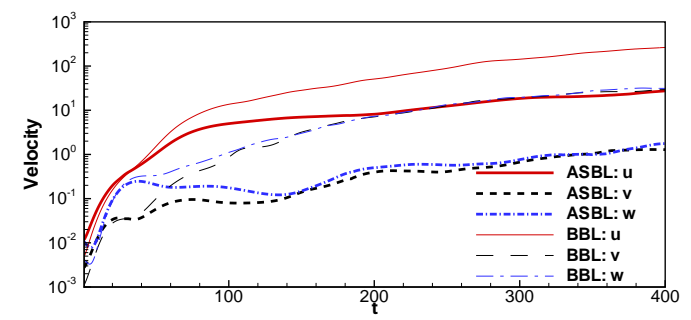

(a)

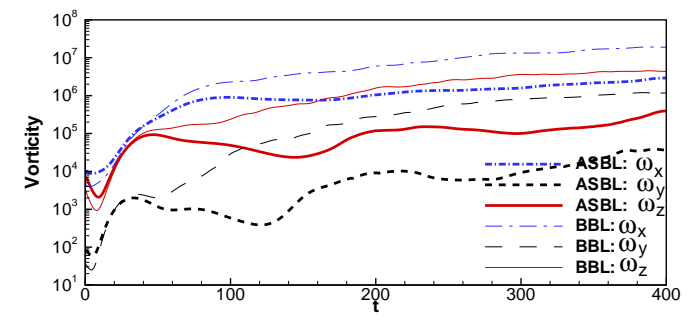

(b)

Figure 20: (Color online) Evolution in time of the rms values of the three components of velocity (solid lines for $u^{\prime}$, dashed for $v^{\prime}$, dashed-dotted for $w^{\prime}$ ) (a) and vorticity (solid lines for $\omega_{z}^{\prime}$, dashed for $\omega_{y}^{\prime}$, dashed-dotted for $\omega_{x}^{\prime}$ ) (b) for a DNS initialized by the selected NLOP obtained for the $\mathrm{BBL}$, injected into the ASBL (thick lines) and the BBL (thin lines) flows. .

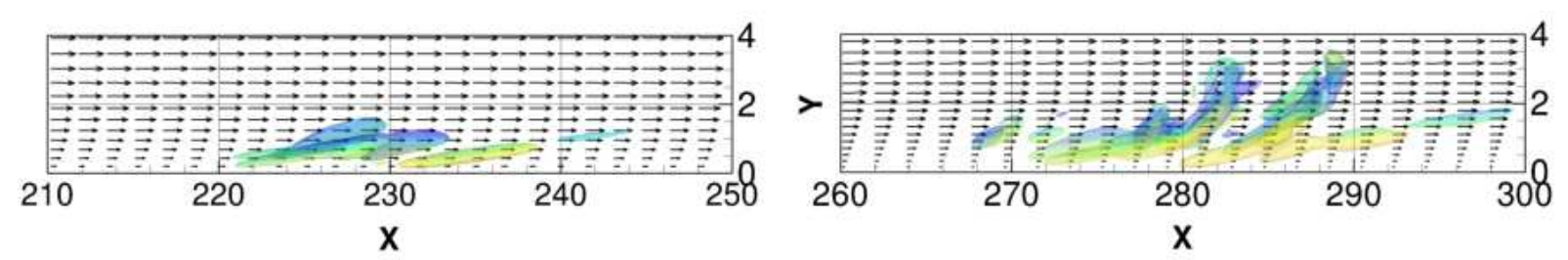

Figure 21: (Color online) Snapshots of the evolution in time of the selected NLOP obtained for the $\mathrm{BBL}$ at $R e=610$, injected in the ASBL (left frame) and the BBL flow (right frame): isosurfaces of the Q-criterion for $t=100$ and vectors of the base flow.

This conjecture can be verified by visualizing the activation of the transport terms of the spanwise vorticity (characterizing the head of the hairpin) by the mean flow. Figure 22 shows one of these two transport terms, $U v_{x}^{\prime}$, for the BBL (top frames) and the ASBL (bottom frames) at $t=100$ (left frames) and $t=150$ (right frames). It appears that, for the BBL, the terms is activated at the head of the hairpin vortices, spreading in space at larger time. Whereas, for the ASBL, they are characterized by a lower amplitude (lower than half the one for the BBL), and they are rapidly damped in time. This confirms that the transport of the spanwise vorticity by the mean flow is indeed the mechanism which can explain the large differences in the symmetry of the non linear optimal perturbations. Such a result shows that it is crucial to take into account the non linearity as well as the non-parallelism of a flow at the same time, for determining with accuracy the most effective 

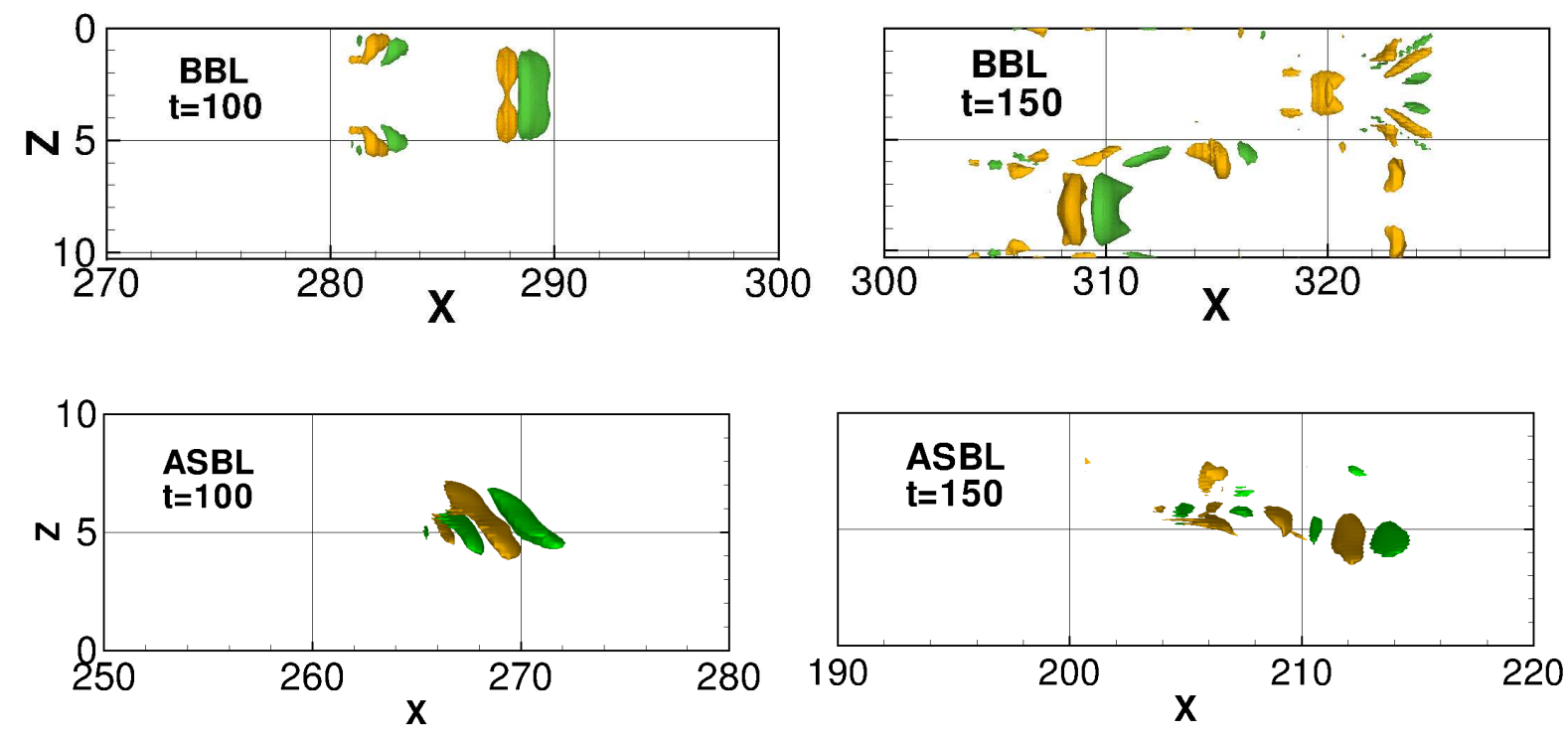

Figure 22: (Color online) Snapshots of the evolution in time of the advection term $U v^{\prime}{ }_{x}$ obtained for the BBL (top frames) and the ASBL (bottom frames) at $t=100$ (left frames) and $t=150$ (right frames): isosurfaces $U v^{\prime}{ }_{x}= \pm 0.13$ for the BBL, $U v^{\prime}{ }_{x}= \pm 0.06$ for the ASBL.

route to transition.

For larger Reynolds numbers, since the suction velocity is much weaker, the velocity profile tends to be closer to that of the BBL flow. Thus, the transition scenario tends to become similar to the BBL one, generating an hairpin vortex at small times, for symmetrical initial perturbations. Figure 23 shows three snapshots of the evolution in time of the NLOP obtained for $R e=5000$ and $E_{0}=4.25 \times 10^{-9}$, showing a behaviour very similar to the one recovered for the BBL in figure 13. In particular, one can observe the formation of $\Lambda$ structures for the negative streamwise velocity component and the streamwise vorticity. Figure 24 shows the generation of a train of hairpin vortices at $t=75$ and $=100$, as well as the base flow profiles, which appear much less full than in the previous case. This confirms that wall suction is a crucial parameter for the stabilisation of a boundary layer flow, since, when it is strong enough, it can modify the base flow profile and delay the formation of strongly growing vortical structures such as the hairpin vortices. 

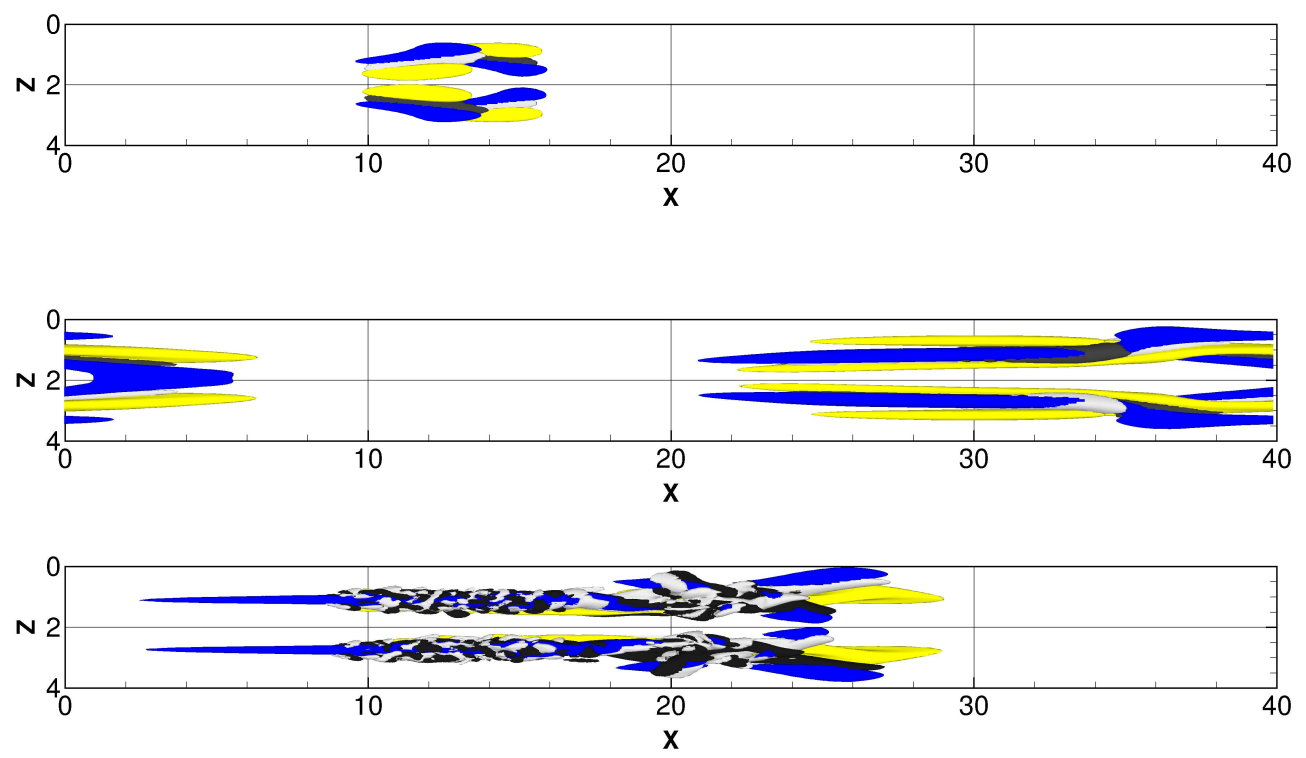

Figure 23: (Color online) Snapshots of the evolution in time of the symmetrical NLOP at $R e=$ 5000, with initial energy $E_{0}=4.25 \times 10^{-9}$, extracted at $t=50,100,150$, from top to bottom. The isosurfaces represent the streamwise velocity and vorticity perturbations (yellow and blue, for $u^{\prime}= \pm 0.015,0.03,0.1$, from top to bottom; black and white, $\omega^{\prime}{ }_{x}= \pm 0.15,0.1,0.25$, from top to bottom).

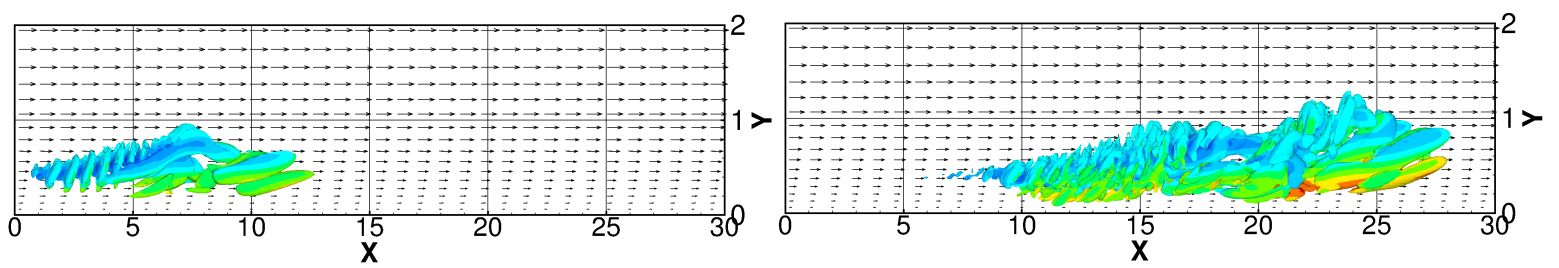

Figure 24: (Color online) Snapshots of the evolution in time of the selected NLOP obtained for the ASBL at $R e=5000$, extracted at $t=75$ (left frame) and $t=100$ (right frame): isosurfaces of the Q-criterion $(Q=20)$ and vectors of the base flow.

\section{SUMMARY}

A variational procedure has been employed to find non linear optimal disturbances in the asymptotic suction boundary layer (ASBL) flow. These perturbations are defined as the ones 
yielding the largest energy growth at a given target time $T$, for a given Reynolds number Re. The results have been compared with those obtained using the same approach in the case of the Blasius boundary layer (BBL) flow [8]. The influence of the different structure of the ASBL velocity profile with respect to the BBL on the non-linear optimal growth mechanism has been studied. It has been found that suction remarkably reduces the optimal energy gain in the non linear case. Moreover, the optimal perturbation obtained in the present case shares the same basic structure found for different shear flows such as the BBL and the Couette flows. However, unlike the BBL case, the optimal perturbation for the ASBL flow for low to moderate Reynolds number is not spanwise-symmetric. In particular, it has been found that a value of the Reynolds number exists between 5000 and 10000 for which the non linear optimal perturbation changes from a non symmetric shape to a symmetric one. Therefore, for sufficiently high Reynolds numbers (low suction velocity), the structure of the non linear optimal disturbance becomes similar to the one of the BBL. By bisecting the initial energy of the non linear optimal perturbations, minimal energy thresholds for subcritical transition to turbulence have been obtained. These energy thresholds are found to be 1 to 4 order of magnitude lower than the ones found in other transition scenarios such as secondary instability of elongated streamwise vortices, random noise, oblique waves and localized streamwise-aligned disturbances [33]. For $610<R e<5000$, these thresholds are found to scale as $R e^{-2}$, suggesting a new scaling law for transition in the ASBL.

Finally, direct numerical simulations show that the different structure of the base flow with respect to the $\mathrm{BBL}$ leads to a different evolution of the initial perturbation. In fact, unlike the case of the BBL flow, for low to moderate values of the Reynolds number, the formation of hairpin vortices is not observed in the transition process before break-up to turbulence, and a sinuous transition scenario is observed. This appears to be due to the lower tilting of the vortices induced by the fuller velocity profile in the ASBL case, which delay the formation of hairpin vortices. However, when wall suction is not strong enough (i.e., at large Re, for a given freestream velocity) the vorticity transport and tilting mechanism giving birth to the hairpin vortex is observed again. Future work will aim at investigate the existence of a similar change of symmetry in the non-linear optimal perturbations for different shear flows such as the plane Poiseuille flow, as well as the influence of the independent parameters of the optimisation, such as the initial energy and the target time, on the optima perturbation structure. 


\section{Acknowledgments}

Some computations have been performed on the Power 6 of the IDRIS, France.

[1] Anderson, J. D. JR 2005 Ludwig prandtl's boundary layer. Physics Today 58, 42-48.

[2] Andersson, P., Berggren, M. \& S., Henningson D. 1999 Optimal disturbances and byoass transition in boundary layers. Phys. Fluids 11 (1), 134-150.

[3] Andersson, P., Brandt, L., Bottaro, A. \& Henningson, D. S. 2001 On the breakdown of boundary layer streaks. J. Fluid Mech. 428, 29-60.

[4] Brandt, L., Schlatter, P. \& Henningson, D. S. 2004 Transition in a boundary layers subject to free-stream turbulence. J. Fluid Mech. 517, 167-198.

[5] Butler, K. M. \& Farrell, B. F. 1992 Three-dimensional optimal perturbations in viscous shear flow. Phys. Fluids A 4, 1637-1650.

[6] Bystrom, M.G., Levin, O. \& Henningson, D. S. 2007 Optimal disturbances in suction boundary layers . Eur. J. Mech. B/Fluids 26, 330-343.

[7] Cherubini, S. \& De Palma, P. 2014 Minimal perturbations approaching the edge of chaos in a Couette flow . Fluid Dynamics Research 46, 041403.

[8] Cherubini, S., De Palma, P., Robinet, J.-Ch. \& Bottaro, A. 2010 Rapid path to transition via nonlinear localized optimal perturbations . Phys. Rev. E 82, 066302.

[9] Cherubini, S., De Palma, P., Robinet, J.-Ch. \& Bottaro, A. 2011 Edge states in a boundary layer. Phys. of Fluids 23, 051705.

[10] Cherubini, S., De Palma, P., Robinet, J.-Ch. \& Bottaro, A. 2011 The minimal seed of turbulence transition in a boundary layer . J. Fluid Mech. 689, 221-253.

[11] Cherubini, S., De Palma, P., Robinet, J.-Ch. \& Bottaro, A. 2012 A purely nonlinear route to transition approaching the edge of chaos in a boundary layer . Fluid Dynamics Research 44, 031404 .

[12] Cherubini, S. \& Palma, De 2012 Nonlinear optimal perturbations in a Couette flow: bursting and transition. J. Fluid Mech. 716, 251-279.

[13] Cherubini, S., Robinet, J.-Ch., Bottaro, A. \& De Palma, P. 2010 Optimal wave packets in a boundary layer and initial phases of a turbulent spot . J. Fluid Mech. 656, 231- 
259.

[14] Cherubini, S., Robinet, J.-Ch. \& De Palma, P. 2013 Nonlinear control of unsteady finiteamplitude perturbations in the Blasius boundary-layer flow . J. Fluid Mech. 737, 440-465.

[15] Corbett, P. \& Bottaro, A. 2000 Optimal perturbations for boundary layers subject to stream-wise pressure gradient . Phys. Fluids 12, 120-130.

[16] Duguet, Y., Monokrousos, A., Brandt, L. \& Henningson, D. S. 2013 Minimal transition thresholds in plane Couette flow. Phys. Fluids 25, 084103.

[17] Eckhardt, B., Schneider, T. M., Hof, B. \& Westerweel, J. 2007 Turbulence transition of pipe flow . Annu. Rev. Fluid Mech. 39, 447-468.

[18] FARrell, B. 1988 Optimal excitation of perturbations in viscous shear flow . Phys. Fluids 31, 2093-2102.

[19] Fransson, J.H.M., Matsubara, M. \& Alfredsson, P. H. 2005 Transition induced by free stream turbulence. J. Fluid Mech. 527 (10), 1-25.

[20] Fransson, J. H. M. \& Alfredsson, P. H. 2003 On the disturbance growth in an asymptotic suction boundary layers. J. Fluid Mech. 482, 51-90.

[21] Fransson, J. H. M. \& Corbett, P. 2003 Optimal linear growth in the asymptotic suction boundary layer. Eur. J. Mech./Fluids 22, 259-270.

[22] Griffith, A. A. \& Meredith, F. W. 1936 The possible improvement in aircraft performance due to boundary layer suction. Rep. Aero. Res. Coun. Tech. Rep.

[23] Hocking, L. M. 1975 Non-linear instability of the asymptotic suction velocity profile . Q. J. Mech. Appl. Maths 28, 341-353.

[24] Hoepffner, J., Brandt, L. \& Henningson, D. S. 2005 Transient growth on boundary layer streaks. J. Fluid Mech. 537, 91-100.

[25] Hof, B., van Doorne, C.W.H., Westerweel, J., Nieuwstadt, F.T.M., Faisst, H., Eckhardt, B., Wedin, H., Kerswell, R.R. \& Waleffe, F. 2004 Experimental Observation of Nonlinear Traveling Waves in Turbulent Pipe Flow . Science 305, 1594-1598.

[26] Joslin, R. D. 1998 Aircraft Laminar Flow Control . AIAA Paper 97, 0625.

[27] Kerswell, R. R., Pringle, C. C. T. \& Willis, A. P. 2014 An optimisation approach for analysing nonlinear stability with transition to turbulence in fluids as an exemplar . Rep. Prog. Phys. 77, 085901.

[28] Khapko, T., Duguet, Y., Kreilos, T., Schlatter, P., Eckhardt, B. \& Henningson, 
D. 2014 Complexity of localised coherent structures in a boundary-layer flow . The European Physical Journal E 37, 1-12.

[29] Khapko, T., Kreilos, T., Schlatter, P., Duguet, Y., Eckhardt, B. \& Henningson, D. 2013 Localized edge states in the asymptotic suction boundary layer . J. Fluid Mech. 717, R6.

[30] Klebanoff, P. S. 1971 Effects of freestream turbulence on the laminar boundary layer . Bull Am. Phys. Soc. 10 (1323).

[31] Kreilos, T., Veble, G., Schneider, T. M. \& Eckhardt, B. 2013 Edge states for the turbulence transition in the asymptotic suction boundary layer . J. Fluid Mech. 726, 100-122.

[32] Landahl, M. T. 1980 A note on an algebraic instability of inviscid parallel shear flows. J. Fluid Mech. 98, 243-251.

[33] Levin, O., Davidsson, N. \& Henningson, D. S. 2005 Transition thresholds in the asymptotic suction boundary layer . Physics of Fluids. A, Fluid Dynamics 17, 114104.

[34] Levin, O. \& Henningson, D. S. 2007 Turbulent spots in the asymptotic suction boundary layer. J. Fluid Mech 548, 397-413.

[35] Luchini, P. 2000 Reynolds number indipendent instability of the Blasius boundary layer over a flat surface: optimal perturbations. J. Fluid Mech. 404, 289-309.

[36] Luchini, P. \& Bottaro 2014 Adjoint Equations in Stability Analysis . Ann. Rev. Fluid Mech. 46, 493-517.

[37] Matsubara, M. \& Alfredsson, P.H. 2001 Disturbance growth in boundary layers subjected to free-stream turbulence. J. Fluid Mech. 430, 149-168.

[38] Monokrousos, A., Bottaro, A., Brandt, L., Di Vita, A. \& Henningson, D. S. 2011 Non-equilibrium thermodynamics and the optimal path to turbulence in shear flows. Phys. Rev. Lett. 106, 134502.

[39] Nagata, M. 1990 Three-dimensional finite amplitude solutions in plane Couette flow . J. Fluid Mech. 217, 519-527.

[40] Pringle, C. C. T. \& Kerswell, R.R. 2010 Using nonlinear transient growth to construct the minimal seed for shear flow turbulence. Phys. Rev. Lett. 105, 154502.

[41] Pringle, C. C. T., Willis, A. \& Kerswell, R.R. 2011 Minimal seeds for shear flow turbulence: using nonlinear transient growth to touch the edge of chaos . J. Fluid Mech. 702, 415-443. 
[42] Rabin, S.M.E., Caulfield, C. P. \& Kerswell, R.R. 2012 Triggering turbulence efficiently in plane Couette flow . J. Fluid Mech. 712, 244-272.

[43] Reddy, S. C. \& Henningson, D. S. 1993 Energy growth in viscous channel flows . J. Fluid Mech. 252, 209-238.

[44] Schlichting, H. 2004 Boundary layer theory . Springer.

[45] Schneider, T. M., Eckhardt, B. \& Yorke, J.A. 2007 Turbulence Transition and the Edge of Chaos in Pipe Flow . Phys. Rev. Lett. 99, 034502.

[46] Schneider, T. M., Gibson, J. F., Lagha, M., De Lillo, F. \& Eckhardt, B. 2008 Laminar-turbulent boundary in plane Couette flow . Phys. Rev. E 78, 037301.

[47] Schoppa, W. \& Hussain, F. 2002 Coherent structure generation in near-wall turbulence . J. Fluid Mech. 453, 57-108.

[48] Skufca, J. D., Yorke, J.A. \& Eckhardt, B. 2006 Edge of chaos in a parallel shear flow . Phys. Rev. Lett. 96, 174101.

[49] Verzicco, R. \& Orlandi, P. 1996 A finite-difference scheme for the three-dimensional incompressible flows in cylindrical coordinates . J. Comp. Phys. 123 (2), 402-414.

[50] Viswanath, D. \& Cvitanovic, P. 2009 Stable manifolds and the transition to turbulence in pipe flow . J. Fluid Mech. 627, 215.

[51] Waleffe, F. 1997 On a self-sustaining process in shear flows . Phys. Fluids 9, 883-901.

[52] Waleffe, F. 1998 Three-dimensional states in plane shear flow . Phys. Rev. Lett. 81, 41404143.

[53] Wedin, H. \& Kerswell, R.R. 2004 Exact coherent structures in pipe flow: travelling wave solutions. J. Fluid Mech. 508, 333-371.

[54] Yoshioka, S., Fransson, J. H. M. \& Alfredsson, P. H. 2004 Free stream turbulence induced disturbances in boundary layers with wall suction . Phys. Fluids 16, 3530.

[55] Zuccher, S., Luchini, P. \& Bottaro, A. 2004 Algebraic growth in a blasius boundary layer: optimal and robust control by mean suction in the nonlinear regime. Eur. J. Mech. B/Fluids 513, 135-160. 